\title{
Genome-wide transcriptional analysis of grapevine berry ripening reveals a set of genes similarly modulated during three seasons and the occurrence of an oxidative burst at vèraison
}

\author{
Stefania Pilati1 ${ }^{1}$ Michele Perazzolli1,4, Andrea Malossini ${ }^{2}$, \\ Alessandro Cestaro ${ }^{1}$, Lorenzo Demattè ${ }^{1,3}$, Paolo Fontana ${ }^{1}$, Antonio Dal Ri ${ }^{1}$, \\ Roberto Viola1 ${ }^{1}$, Riccardo Velasco ${ }^{1}$ and Claudio Moser*1
}

\author{
Address: ${ }^{1}$ Department of Genetics and Molecular Biology; IASMA Research Center, Via E. Mach 1, 38010 S. Michele a/Adige (TN), Italy, \\ ${ }^{2}$ Department of Information and Communication Technology, University of Trento, Via Sommarive 14, 38050 Povo (TN), Italy, ${ }^{3}$ Lorenzo \\ Demattè: Microsoft Research-University of Trento Centre, Piazza Manci 17, 38050 Povo (TN) Italy and ${ }^{4}$ Michele Perazzolli: SafeCrop Centre, \\ Istituto Agrario San Michele a/Adige, Via E. Mach 1, 38010 S. Michele a/Adige (TN), Italy \\ Email: Stefania Pilati - stefania.pilati@iasma.it; Michele Perazzolli - michele.perazzolli@iasma.it; Andrea Malossini - malossin@dit.unitn.it; \\ Alessandro Cestaro - alessandro.cestaro@iasma.it; Lorenzo Demattè - dematte@cosbi.eu; Paolo Fontana - paolo.fontana@iasma.it; Antonio Dal \\ Ri - antonio.dalri@iasma.it; Roberto Viola - roberto.viola@iasma.it; Riccardo Velasco - riccardo.velasco@iasma.it; \\ Claudio Moser* - claudio.moser@iasma.it \\ * Corresponding author
}

Published: 22 November 2007

BMC Genomics 2007, 8:428 doi:10.1 I86/I47I-2164-8-428
Received: 10 August 2007

Accepted: 22 November 2007

This article is available from: http://www.biomedcentral.com//47I-2164/8/428

(c) 2007 Pilati et al; licensee BioMed Central Ltd.

This is an Open Access article distributed under the terms of the Creative Commons Attribution License (http://creativecommons.org/licenses/by/2.0), which permits unrestricted use, distribution, and reproduction in any medium, provided the original work is properly cited.

\begin{abstract}
Background: Grapevine (Vitis species) is among the most important fruit crops in terms of cultivated area and economic impact. Despite this relevance, little is known about the transcriptional changes and the regulatory circuits underlying the biochemical and physical changes occurring during berry development.

Results: Fruit ripening in the non-climacteric crop species Vitis vinifera $L$. has been investigated at the transcriptional level by the use of the Affymetrix Vitis GeneChip ${ }^{\circledR}$ which contains approximately 14,500 unigenes. Gene expression data obtained from berries sampled before and after véraison in three growing years, were analyzed to identify genes specifically involved in fruit ripening and to investigate seasonal influences on the process. From these analyses a core set of 1477 genes was found which was similarly modulated in all seasons. We were able to separate ripening specific isoforms within gene families and to identify ripening related genes which appeared strongly regulated also by the seasonal weather conditions. Transcripts annotation by Gene Ontology vocabulary revealed five overrepresented functional categories of which cell wall organization and biogenesis, carbohydrate and secondary metabolisms and stress response were specifically induced during the ripening phase, while photosynthesis was strongly repressed. About $19 \%$ of the core gene set was characterized by genes involved in regulatory processes, such as transcription factors and transcripts related to hormonal metabolism and signal transduction. Auxin, ethylene and light emerged as the main stimuli influencing berry development. In addition, an oxidative burst, previously not detected in grapevine, characterized by rapid accumulation of $\mathrm{H}_{2} \mathrm{O}_{2}$ starting from véraison and by the modulation of many ROS scavenging enzymes, was observed.
\end{abstract}




\begin{abstract}
Conclusion: The time-course gene expression analysis of grapevine berry development has identified the occurrence of two well distinct phases along the process. The pre-véraison phase represents a reprogramming stage of the cellular metabolism, characterized by the expression of numerous genes involved in hormonal signalling and transcriptional regulation. The post-véraison phase is characterized by the onset of a ripening-specialized metabolism responsible for the phenotypic traits of the ripe berry. Between the two phases, at véraison, an oxidative burst and the concurrent modulation of the anti-oxidative enzymatic network was observed. The large number of regulatory genes we have identified represents a powerful new resource for dissecting the mechanisms of fruit ripening control in non-climacteric plants.
\end{abstract}

\section{Background}

Grape is among the most ancient, widely cultivated fruit crops. The keen interest in the understanding of grape berry ripening is justified by the economic relevance of the quality of grapes and their processed products, such as wine, juice and dried fruit. The onset of the genomic era has brought unprecedented progress into our knowledge of Vitis molecular biology, and powerful genetic and genomic tools are now available or are being developed, such as mapping populations, genetic and physical maps $[1,2]$, an extensive ESTs collection $(342,576$ sequences deposited at the NCBI EST database-March 19, 2007, [3]) and several tools for gene expression analysis. These resources enable us to identify grape as a model plant for studies on non-climacteric anthocyanin accumulating fruits, beside tomato, which is currently the model plant for climacteric carotenoid accumulating fruits. Grape berry development from anthesis to ripening is classically divided into three phases on the basis of chemical and morphological traits [4]: berry formation, characterized by exponential growth of the berry and accumulation of organic acids, mainly malate, in the vacuole; véraison, a transition phase during which growth declines and berries start to change colour and soften; ripening, characterized by an increase in $\mathrm{pH}$, marked berry growth and accumulation of sugars, anthocyanins and flavour-enhancing compounds. Published studies based on EST analysis [5-7], differential display [8], cDNA-arrays [9] and oligo arrays [10] have shown distinct and extensive changes in the transcriptome occurring during ripening. These studies report up to 740 developmentally regulated genes mainly involved in defence, stress response, primary and secondary metabolism, berry growth and hormonal metabolism. The very recent study of Grimplet et al. [11], provides an extensive description of these functional classes investigating their tissue-specific expression in the ripe berry by means of the Affymetrix Vitis GeneChip ${ }^{\circledast}$.

With the aim of understanding the transcriptional changes underlying grapevine berry ripening and the regulatory mechanisms acting at this level, we were able to identify 1477 genes modulated during berry development, comprising 282 regulatory factors in addition to the structural genes, by the use of the Affymetrix Vitis GeneChip ${ }^{\circledast}$. The results, obtained from replicated experiments carried out in three growing seasons, provide also insights into the relationship between genetic and environmental effects on berry development control.

\section{Results and Discussion Grape berries phenotypic evaluations}

Figure 1 shows the changes in average berry weight, organic acids, total soluble solids and anthocyanin content of grape berries sampled weekly during the 2003, 2005 and 2006 growing seasons. The date of véraison was set at approximately 8 weeks post-flowering (wpf) in all years. In order to characterize berry ripening, we chose three distinct moments according to [12]: the last stage of berry formation, time-point A (TP A, stage E-L 33), characterized by small hard green berries still accumulating organic acids; and two stages during berry ripening, TP B (stage E-L 34), just before véraison, characterized by berries in the green state with maximum acidic content starting to soften and TP C (stage E-L 36), when the ripening process is well established and berries are growing fast, colouring and accumulating sugars. The identification of the corresponding developmental stages in the three years was based on the biochemical profiles. As average temperatures during 2003 remained constantly unusually higher from April to October [see Additional file 1], flowering and harvest occurred approximately one week earlier in 2003.

\section{Statistical analysis}

RNA extracted in triplicate from berries harvested at three time points was used to hybridize 27 Affymetrix Vitis GeneChips ${ }^{\circledast}$. Datasets from each season were analyzed separately. The quality of the biological replicates was evaluated by the R-squared coefficient [see Additional file 2], which ranged from 0.89 to 0.99 . Minimum R-squared values were found for TP B samples, suggesting a higher sample heterogeneity approaching véraison, when dramatic transcriptional changes occur in less than 48 hours [10]. 


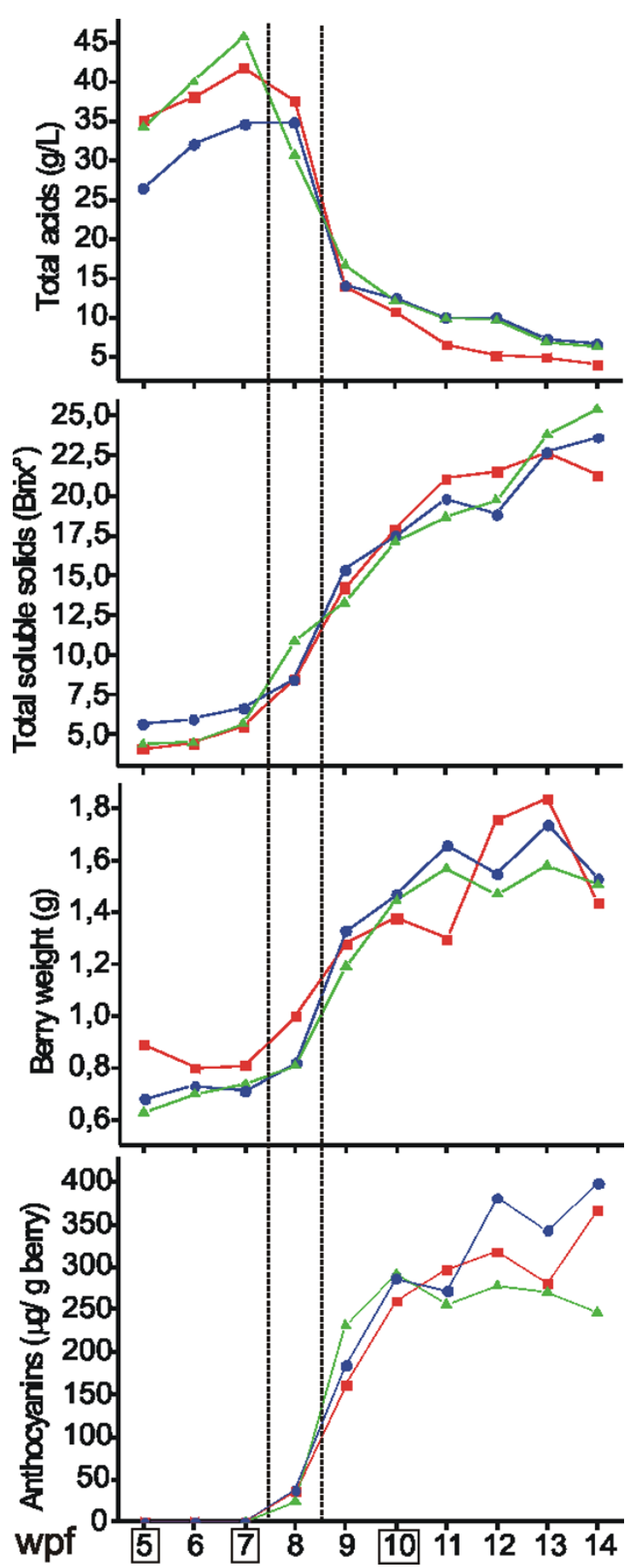

Figure I

Biochemical changes observed during Pinot Noir berry development in 2003 (red), 2005 (blue) and 2006 (green). Total acids (expressed as grams of tartaric acid per liter of must), total soluble solids, and anthocyanins content and berry weight averaged on a pool of 50 berries are reported. The dashed area indicates véraison. The weeks post flowering (wpf) corresponding to the samples used for the hybridization experiments are enclosed in boxes.
Principal Component Analysis (PCA) confirmed the uniformity of the biological replicates as the nine groups of replicates clusterised tightly (Fig. 2). The greatest variance in gene expression was found between samples from the three growing seasons, as they were separated along the first component ( $51 \%$ of the total variance). In particular, the largest difference was evident between the 2003 expression data compared to the 2005 and 2006, suggesting that the environmental conditions greatly affect the berry transcriptome. [see Additional file 1]. On the other hand, the second principal component (24\% of the total variance) separated neatly the pre-véraison stages (TP A and TP B) from the post-véraison one (TP C), confirming previous observations of extensive transcriptional changes occurring from berry formation to berry ripening [10].

Class comparison analysis by means of Significance Analysis of Microarray [13], imposing a minimum fold change of 2 in at least one comparison (TP A vs. TP B and TP B vs. TP C), produced three sets of 2632, 4238 and 3856 differentially expressed probesets for the 2003, 2005 and 2006 samples, respectively. The size difference between the three sets likely reflects inter-seasonal biological differences. The comparison of the three datasets identified a common set of 1700 modulated transcripts and among these, 1477 transcripts with a conserved profile in the

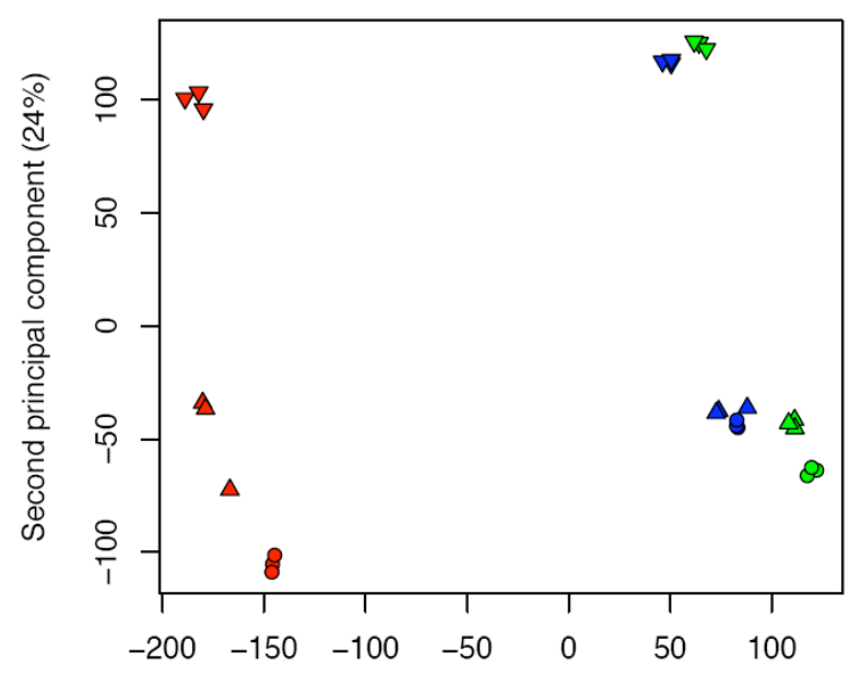

First principal component (51\%)

Figure 2

Principal component analysis (PCA) of the 27 expression datasets. Scaled expression data relative to 2003 (red), 2005 (blue) and 2006 (green) samples are sharply separated according to the sampling year (first principal component) and to the developmental stage (second principal component). Biological replicates relative to time point $A, B$ and $C$ are represented as circles, triangles and downwards triangles, respectively. 
three years (Pearson's correlation coefficient $>0.5$ in any pairwise comparison). This 1477 core dataset, which corresponds to $9 \%$ of the chip probesets, is listed in Additional file 3. It represents a highly conserved expression network reasonably involved in an internal basic functional program, such as berry ripening.

\section{Annotation of the differentially expressed genes}

Automatic annotation of all the Vitis GeneChip ${ }^{\circledR}$ sequences was performed using the Gene Ontology (GO) classification [14]. The core set of 1477 differentially expressed genes was subsequently manually checked and integrated with additional GO 'biological process' terms. Transcripts were then grouped into 17 GO functional categories [see Additional file 3 and 4] whose distribution among the entire chip and the core set was statistically compared (Fig. 3). Nine categories were differentially represented at significant levels during berry development and five of these were overrepresented in the regulated gene core set. These were "carbohydrate metabolism", "cell wall organization and biogenesis," "response to stimulus," "secondary metabolism" and "photosynthe- sis," all processes that ought to be specifically regulated during grape berry development. The four underrepresented categories were "nucleobase, nucleoside, nucleotide and nucleic acid metabolism," "protein biosynthesis," "protein metabolism" and "transport." These classes may either play a minor role in the developmental program or their involvement could be restricted to a small subset of genes.

The functional class distribution of the Vitis GeneChip ${ }^{\circledR}$ was also compared to that of the Arabidopsis genome available at TAIR ([15], [Additional file 5]). The overall agreement in the class distributions confirms the Vitis GeneChip ${ }^{\circledR}$ as a reliable toolkit for the investigation of the grape expressed genome.

\section{Cluster analysis and functional categories modulation during berry ripening}

Cluster analysis of the gene core set was based on the kmeans method using Pearson's correlation distance calculated on the gene expression profiles. Transcripts were divided into eight groups representing the minimum

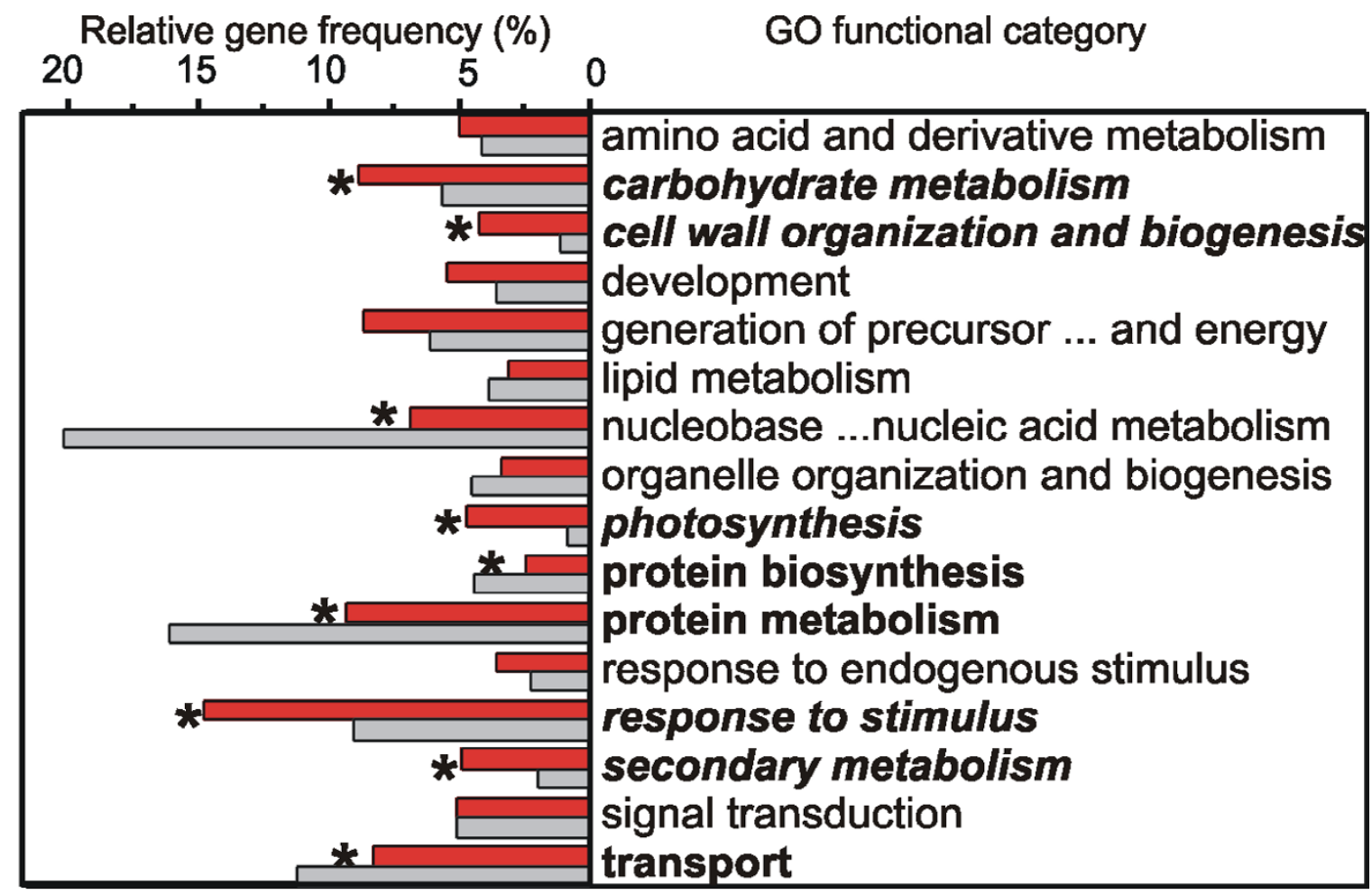

Figure 3

Functional categories distribution in the core set of the modulated genes (red) and in the entire Vitis GeneChip $^{\circledR}$ (grey). Frequencies are calculated as percentage of the whole number of 'GO biological process' terms ( 1,825 and 21,890 in the modulated and chip sets, respectively). Nine classes, marked by an asterisk and written in bold, resulted differently represented in the modulated set compared to the entire chip after statistical analysis ( $p$-value $<0.00 \mathrm{I}$ ). In italics are the five categories overrepresented in the modulated set. 
number of profiles that could be obtained with three time-points. We observed good agreement between clustering in the three gene sets $(80 \%$ of the transcripts fell in the same cluster in all seasons). We decided to use the 2005 expression data for the cluster representation (Fig. 4 ), due to the smaller variance among replicates [see Additional file 2]. As expected from PCA, the two most populated clusters were number 4 and 8 , composed by genes modulated positively and negatively from TP B to TP C, respectively. Conversely, clusters 2 and 6 , consisting of genes modulated only during the pre-véraison interval, were the less populated ones. Approximately the same number of genes was positively or negatively modulated along the whole study interval (cluster 3 and 7). Genes specifically induced around véraison should fall in cluster1 while genes with roles in early and late stages of berry development should fall in cluster 5 .

Functional class distribution frequency was then calculated for each cluster and represented as histogram [Additional file 6]. To better understand the modulation of the functional classes during berry ripening, their frequencies were determined after splitting the experimental interval into two phases: one from TP A to TP B and one from TP $\mathrm{B}$ to TP $\mathrm{C}$. The genes modulated during the first phase derived from clusters 1, 2, 3 (induced) and 5, 6, 7 (repressed), while the genes modulated during the second phase derived from clusters 3, 4, 5 (induced) and 1, 7, 8 (repressed). During the pre-veraison phase, the number of the induced genes (408) exceeded that of the repressed ones (270), while the opposite occurred during the following phase (651 induced vs. 1118 repressed). As depicted in Figure 5, all but two categories showed a general tendency towards an opposite modulation between the first and the second phase. Furthermore in the first phase a general bias towards induction for the GO categories involved in regulatory mechanisms, namely "nucleobase, nucleoside, nucleotide and nucleic acid metabolism" (mainly transcription factors), "response to endogenous stimulus" (mainly hormone metabolism), "signal transduction," and "protein metabolism" was observed. This suggests a strong cell re-programming taking place in berry cells up to véraison. During the second phase, a marked negative regulation is evident for categories involved in cell division, such as "organelle organization and biogenesis" and "protein biosynthesis," and "photosynthesis." This behaviour is coherent with the slowing down of cell replication and the loss of photosynthetic capacity. On the other hand, the classes "amino acid and derivative metabolism," "carbohydrate metabolism," "cell wall organization and biogenesis," "development," "lipid metabolism," "primary" and "secondary metabolism" display a positive regulation. This indicates the prevalence after véraison of metabolic processes involved in cell wall loosening and synthesis, sugar accumulation and synthesis and transport of metabolites responsible of grape colour and flavour. Finally, the class "response to stimulus" appeared induced in both phases, suggesting that throughout the entire berry development process the cell devotes considerable effort to face different kinds of stimuli, presumably including osmotic, oxidative and biotic stress.

\section{Gene regulation of grape berry ripening}

Our study of the berry ripening process identified 125 transcription factors, 65 genes involved in hormone metabolism and response and 92 involved in signal transduction, corresponding to $8.5 \%, 4.4 \%$ and $6.2 \%$ of the regulated core set, respectively. These figures and similar data recently obtained for tomato and Citrus fruit $[16,17]$, suggest that a consistent fraction of the modulated fruit transcriptome is devoted to the control of the developmental program. In this section we present our main contribution to the knowledge of gene regulation during berry ripening in grapevine. A restricted list of selected genes involved in the process and discussed in the text is reported in Table 1 with the specific AffyID, whereas the complete list is provided in part A of Additional file 7.

\section{Hormones metabolism and signalling}

Amongst the genes related to hormone metabolism in the core set, those related to auxin and ethylene were the most represented, followed by those related to abscisic acid and brassinosteroids. Few genes related to cytokinines, gibberellins or jasmonic acid were found. These observations are consistent with our knowledge of the relative importance of these signalling pathways during grape berry development $[18,19]$.

In fruits from both climacteric and non-climacteric species, auxins are known to be more abundant immediately after ovary fertilization and to mediate important signals for the onset of fruit development [20,21]. In grape, it has been generally accepted that indole-3-acetic acid (IAA) content reaches its maximal level just after anthesis and then declines to very low levels in the ripe fruit [22], even if a recent study on Vitis vinifera cv Cabernet Sauvignon did not confirm it [23]. The expression of genes involved in cell wall metabolism (GRIP4), anthocyanin synthesis (CHS and UFGT) and sugar storage (GIN1) and the accumulation of the correlated metabolites were all shown to be retarded by auxin treatments at véraison [19].

In the present study, we have identified a gene homologous to the Arabidopsis amidase AtAMI1, which in vitro synthesizes IAA from indole-3-acetamide [24], and its descending profile during ripening agrees well with the decline in IAA levels observed in Vitis labrusca berries [22]. We have also found a gene homologous to an Arabidopsis IAA-amino acid synthetase which may contribute to IAA 


\section{Cluster 1: 210}

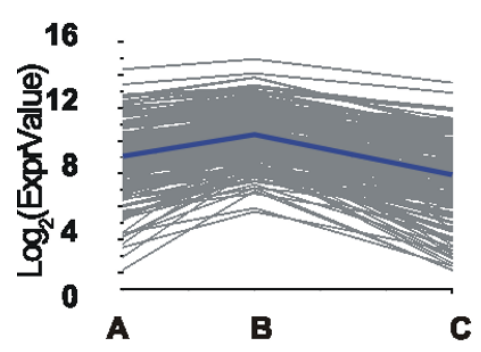

Cluster 2: 22

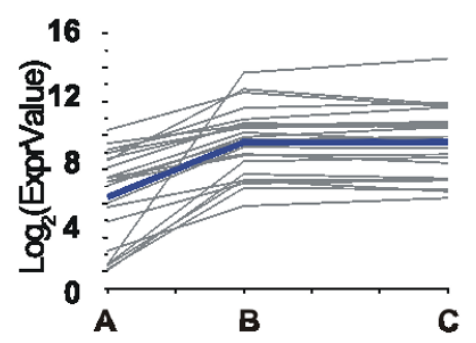

Cluster 3: 127

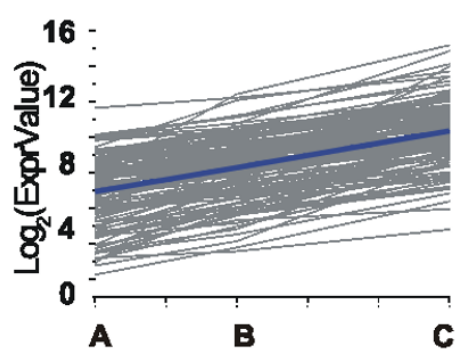

Cluster 4: 335

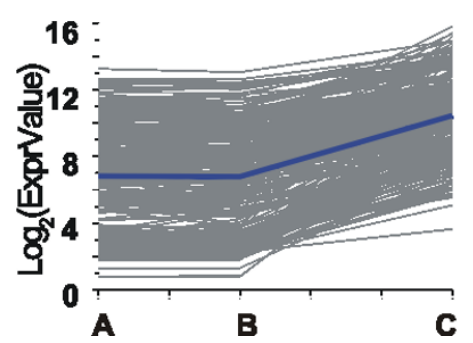

Cluster 5: 76

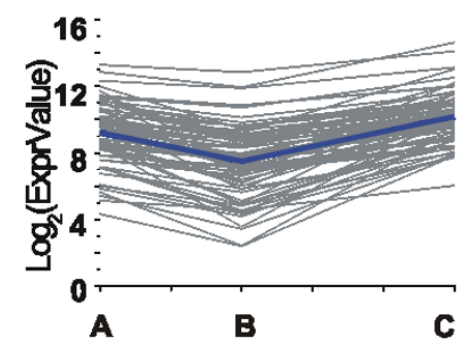

Cluster 6: 21

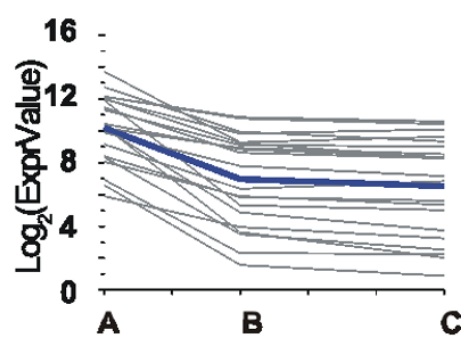

Cluster 7: 128

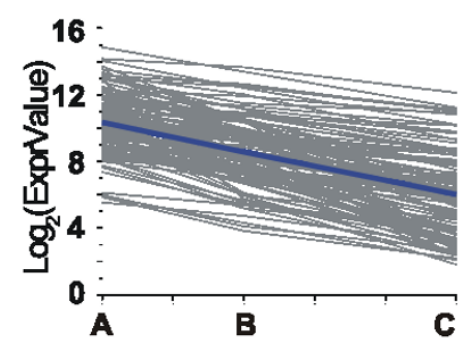

Cluster 8: 558

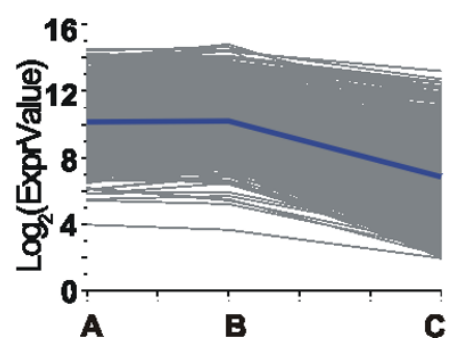

Figure 4

Cluster analysis of the expression profiles of the modulated core set. The expression profiles of the 1477 modulated genes during P. Noir berry ripening were clusterised in eight clusters which represent the minimum number of profiles considering three time points. Clusters were obtained by the k-means method using Pearson's correlation distance. The representative profile and the number of genes in each cluster are indicated. 


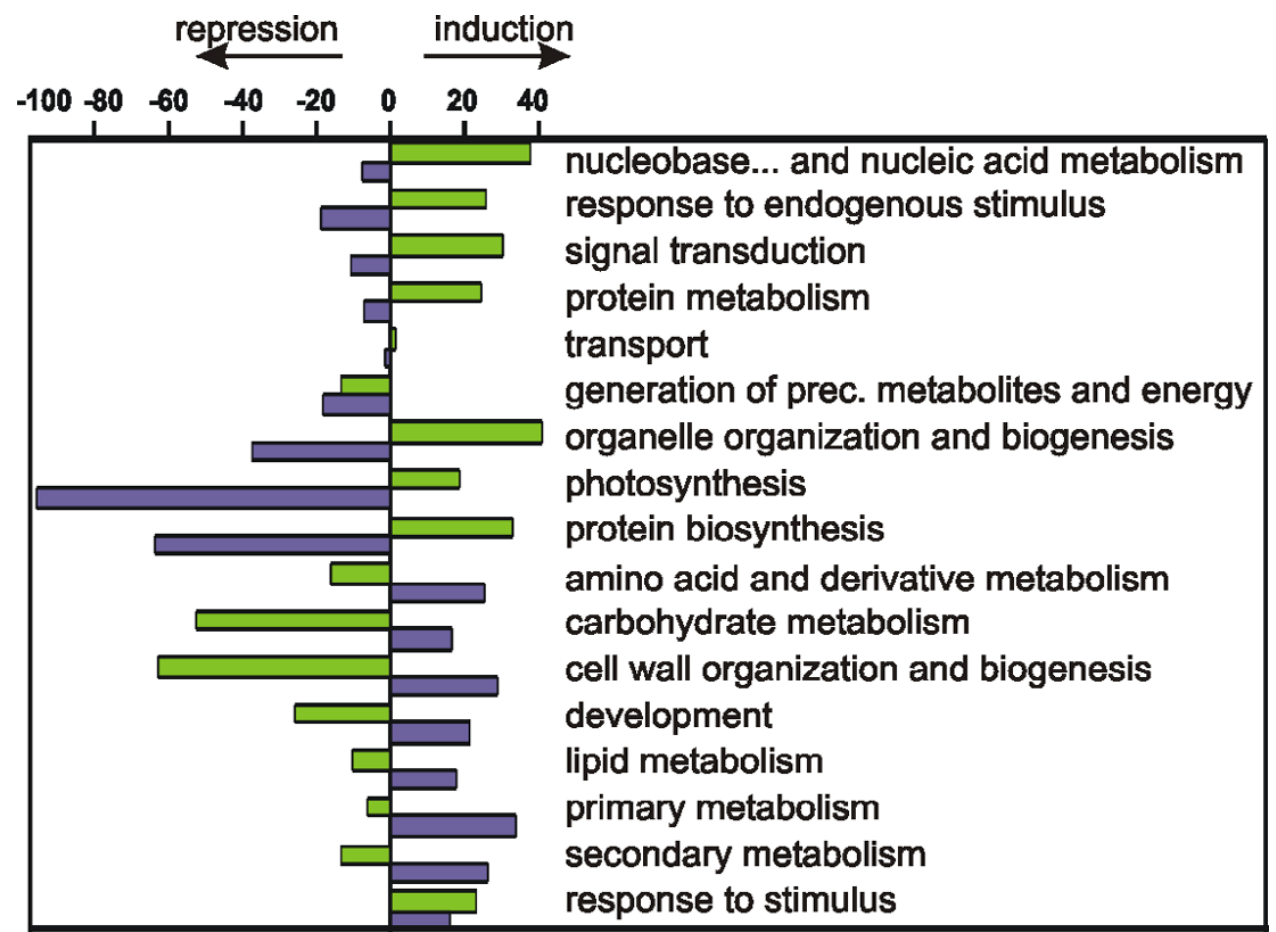

Figure 5

Functional categories distribution before and after-véraison. Induced and repressed functional categories during the first stage (from TP A to TP B, green histograms) derive from clusters I, 2, 3 and 5, 6, 7, respectively, while categories induced and repressed during the second stage (from TP B to TP C, violet histograms) derive from clusters 3, 4, 5 and I, 7, 8, respectively. The relative number of induced $(\mathrm{Ni})$ or repressed $(\mathrm{Nr})$ genes for a specific functional category was calculated with respect to the total number of induced or repressed genes in each stage. The estimate of induction or repression within each functional category was then calculated as follows: estimate of induction/repression $=(\mathrm{Ni}-\mathrm{Nr}) /(\mathrm{Ni}+\mathrm{Nr})$. Positive values represent an overall induction and negative values an overall repression.

intracellular homeostasis via amino acid conjugation of excess IAA [25]. Two auxin carriers (an AUX1-like and a PIN1-like), putatively mediating auxin efflux, were also expressed before véraison.

We detected numerous auxin-regulated genes belonging to the Aux/IAA, Small Auxin-Up RNA and GH3 gene families. These families are characterized by the presence of an Auxin Responsive Element in the promoter region of their members, recognized by specific Auxin Response Factors (ARFs). Two ARFs, ARF5 and ARF18 and an auxin receptor of the ABP family were found expressed during the prevéraison stage and then steeply repressed during ripening. With the exception of two Aux/IAA proteins, which were induced during ripening, all the other transcripts presented profiles with either a peak of expression around véraison or a decreasing profile after it. The auxin responsive genes seem therefore timely co-ordinated with the cellular auxin level, reflecting its prevalent role in the first stages of berry development. We have also detected a gene (1612001_s_at) homologous to a Capsicum chinense GH3 gene, which has been proposed as point of convergence between auxin and ethylene signals in non-climacteric fruit development [26]. While in pepper this GH3 transcript is up-regulated during ripening due to its ethyleneinducibility, our analyses showed a steep repression of the grape homologue after TP B.

The role of ethylene during development and ripening of some non-climacteric fruits was suggested since the 1970 s and has recently been well documented [27-29]. In grape, a small and transient increase of endogenous ethylene production occurs just before véraison, in concomitance with an increase in 1-aminocyclopropane-1-carboxylic acid (ACC) oxidase activity [29]. Our results well suit these reports, as we observed a peak of expression of ACC oxidase around véraison, while ACC synthase and a putative ethylene-forming-enzyme dioxygenase, detected with similar profile also by others [10], were expressed until véraison and then repressed.

We were not able to identify any receptor or kinase involved in ethylene signal transduction in grape, within the modulated gene set. Instead, we detected four tran- 
Table I: Selection of genes putatively involved in the regulation of berry ripening.

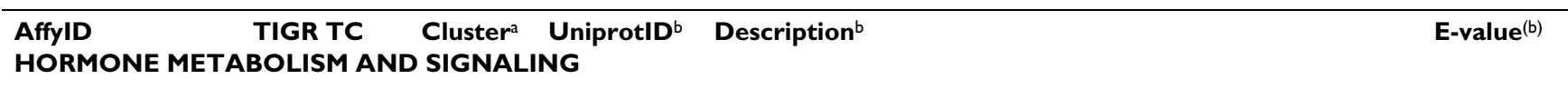

\section{AUXIN}

Auxin synthesis

1610647

Auxin transport

1612060_at

1621946_at

Aux/IAA

1618875_s_at

1617513_at

1615985_at

1614660_at

1621754_at

1615728_at

1611390_a_at

1620512_at

1613468_at

SAUR

I62I20I_at

$\mathrm{GH} 3$

1610880_s_at

$161200 \mid$ s at

1607503_s_at

Other auxin responsive proteins

1619856 at TC45354

1621185_s_at TC45354

1613204_at TC3834 I

1619913_at TC46727

ARF (transcription factor)

1612180_at TC51304

1616225_at TC44210

Auxin receptor

1612090_s_at

TC45I86

ETHYLENE

Ethylene synthesis

1615952_s_at

1622308 at

TC38453

1612699_at

TC4045 I

BQ7986I4

Ethylene responsive transcription factors

1617012_at

TC45046

1611910_s_at

1609990_at

1620278_at

TC39828

TC467I0

1621270_at

TC47575

Ethylene induced genes

1618213_at

1619086_at

$-$

1609780_at

1613123_at

1613177 at

1622850_at

Pathogenesis related genes putatively induced by ethylene

1618835_s_at $\quad$ TC38693 44081228

1622360 at

ABSCISIC ACID

Abscisic acid synthesis

1607029_at TC42536
Abscisic acid responsive proteins

\section{Q9FR37 \\ Amidase (At lg08980 AtAMII)}

Q76DTI

Q8H0E0

Q8LAL2

Q8LAL2

O24542

PI3088

Q84V38

$\mathrm{Q} 84 \mathrm{~V} 38$

Q8RWI6

Q9ZSY8

Q8LSK7

Q6ZKQ7

O22190

Q6QUQ3

Q52QX4

048629

O48629

049235

Q9SV7I

Q6L8T9

Q9C5W9

Q9ZRA4

Q84X67

Q8S933

Q9XIA5

$\mathrm{Q} 8 \mathrm{~S} 9 \mathrm{H} 4$

Q6TKQ3

Q6TKQ3

Q6J9Q4

Q9LYD3

Q9SWV2

Q93W91

Q94E74

Q8S3DI

Q9LSP8

O48631

Q42966

AUX22D

AUX22

Aux/IAA protein

Aux/IAA protein

Aux/IAA protein

Auxin-regulated protein (IAAI)

Auxin-induced protein (SAUR)

auxin-responsive protein

Auxin response factor 5

Auxin response factor 18

ER6 protein (Fragment)

Putative ER6 protein

$\mathrm{PR}-4$ type protein

NtNitrilase 4B (EC 3.5.5.I)
AUXI-like auxin influx carrier protein

PINI-like auxin transport protein

IAA26 (Phytochrome-associated protein I)

IAA26 (Phytochrome-associated protein I)

IAA27 (Phytochrome-associated protein 2)

Indole-3-acetic acid-amido synthetase $\mathrm{GH} 3.3$

Auxin and ethylene responsive $\mathrm{GH} 3$-like protein

Auxin-repressed protein-like protein ARPI

Putative auxin-repressed protein (dormancy)

Putative auxin-repressed protein (dormancy)

2.4-D inducible glutathione S-transferase

Auxin-binding protein $A B P I 9 a$ precursor

I-aminocyclopropane-I-carboxylic acid oxidase I. ACO

I-aminocyclopropane-I-carboxylate synthase. ACC synthase

Similar to ethylene-forming-enzyme-like dioxygenase

Tomato Ethylene response factor I

Putative ethylene response factor ERF3b

Putative ethylene response factor ERF3b

Putative AP2/EREBP transcription factor

Putative AP2/EREBP transcription factor (DREB3)

Putative ER6 protein. Ethylene-responsive protein

bHLH transcription factor (tomato ER33 homolog)

Ethylene-induced calmodulin-binding transcription activator

Ethylene-forming-enzyme-like dioxygenase (tomato E8 homolog)

Putative pathogenesis related protein I precursor
$3,89 \mathrm{E}-152$

2,97E-143

I,05E-5 |

$2,95 \mathrm{E}-63$

$6,04 \mathrm{E}-62$

$2,40 \mathrm{E}-69$

$6,35 \mathrm{E}-60$

$9,19 \mathrm{E}-89$

$0,00 \mathrm{E}+00$

2,92E-98

$9,42 \mathrm{E}-86$

$3,38 \mathrm{E}-99$

5,99E-15

I,78E-33

6,67E-III

$2,50 \mathrm{E}-48$

6,4IE-44

6,4IE-44

I,74E-98

$5,97 \mathrm{E}-116$

I,24E-64

$3,69 \mathrm{E}-88$

I,75E-88

I,20E-167

$3,92 \mathrm{E}-68$

5,03E-29

$5,10 \mathrm{E}-6 \mathrm{I}$

$7,26 \mathrm{E}-124$

I,45E-I 23

2,74E-64

3,34E-63

$3,89 \mathrm{E}-18$

2,55E-5।

5,74E-33

I,04E-6 |

3,76E-94

7,27E-78

I,55E-73

I,62E-80

I,05E-135 
Table I: Selection of genes putatively involved in the regulation of berry ripening. (Continued)

\begin{tabular}{|c|c|c|c|c|c|}
\hline |6182|I_at & TC47430 & 4 & P93615 & ABA induced plasma membrane protein PM 19 & $1,37 E-60$ \\
\hline 1614372 at & TC47836 & 4 & Q6H5X2 & ABA-responsive protein-like & $3,03 \mathrm{E}-6 \mathrm{I}$ \\
\hline 1614788_at & TC45479 & 4 & Q4VT48 & Dehydrin & $2,92 \mathrm{E}-69$ \\
\hline 1621592_s_at & TC45479 & 4 & Q4VT48 & Dehydrin & $2,92 \mathrm{E}-69$ \\
\hline 1606669_s_at & - & 4 & Q5PXH0 & Aquaporin (PIP2_I) & $9,87 \mathrm{E}-74$ \\
\hline 1615808_s_at & TC38I2I & 4 & Q5PXHO & Aquaporin (PIP2_I) & $2,63 \mathrm{E}-162$ \\
\hline 1610982_at & TC38I38 & 8 & O24049 & Major intrinsic protein $\mathrm{C}$, aquaporin & $6,3|E-| 4 \mid$ \\
\hline 1614916_at & TC38138 & 8 & O24049 & Major intrinsic protein $\mathrm{C}$, aquaporin & $6,3|E-| 4 \mid$ \\
\hline 1612244_s_at & TC3828I & 8 & Q9M7B0 & Putative aquaporin PIP2-2 & $2,40 \mathrm{E}-158$ \\
\hline \multicolumn{6}{|c|}{ BRASSINOSTEROIDS } \\
\hline \multicolumn{6}{|c|}{ Brassinosteroid synthesis } \\
\hline 1608099_at & TC40368 & 8 & Q43।47 & Cyt. P450 85AI, C6-oxidase, Dwarf protein & 2,89E-74 \\
\hline \multicolumn{6}{|c|}{ Brassinosteroid receptor } \\
\hline 1612516_at & TC47080 & 1 & Q76FZ8 & Brassinosteroid receptor & $0,00 \mathrm{E}+00$ \\
\hline \multicolumn{6}{|c|}{ GIBBERELLINS } \\
\hline 1610607_at & TC40495 & 5 & Q6NMQ7 & Gibberellin-responsive protein (At Ig74670) & $\mathrm{I}, 04 \mathrm{E}-28$ \\
\hline 1609893_at & TC38517 & 8 & O24040 & LtCORII (Gibberellin regulated protein) & $\mathrm{I}, 62 \mathrm{E}-36$ \\
\hline 1618503_at & TC47765 & 8 & Q75V70 & Gibberellin 2-oxidase I & I,50E-69 \\
\hline \multicolumn{6}{|c|}{ CYTOKININS } \\
\hline 1612955_at & TC47637 & 4 & Q7IBZ3 & Type-A response regulator & 3,73E-II \\
\hline 1619945_at & CB345883 & 8 & Q39636 & CR9 protein. cytokinin-repressed gene & $3,12 \mathrm{E}-30$ \\
\hline 1620306_at & TC47826 & 8 & Q67YU0 & Cytokinin oxidase 5 (cytokinin degradation) & $6,29 \mathrm{E}-113$ \\
\hline \multicolumn{6}{|c|}{ LIGHT STIMULUS } \\
\hline \multicolumn{6}{|l|}{ Circadian rhythm } \\
\hline 1608397_at & TC43962 & 8 & Q6LA43 & Two-component response regulator-like APRR2 (TOC2 protein) & $2,77 E-34$ \\
\hline 1617059_at & TC42407 & 4 & Q9CAV6 & WNKI kinase & $8,07 E-26$ \\
\hline 1609874_at & TC43754 & 8 & Q6R3R2 & CONSTANS-like protein COI & I,80E-76 \\
\hline 1614417_at & TC475II & 3 & O8I834 & Transcription factor Constans-like family (At4g27310) & $\mathrm{I}, 32 \mathrm{E}-32$ \\
\hline 1613978_at & TC49I20 & 3 & Q5GA60 & Putative EARLY flowering 4 protein & $\mathrm{I}, 22 \mathrm{E}-30$ \\
\hline \multicolumn{6}{|l|}{ Flowering control } \\
\hline 1614189 at & TC47473 & 8 & Q76CC3 & Flowering locus $T$ & $1,68 \mathrm{E}-66$ \\
\hline \multicolumn{6}{|c|}{ Transcription factors } \\
\hline 1620877_at & TC40824 & 8 & Q948G4 & Putative GATA-I zinc finger protein & $\mathrm{I}, \mid 4 \mathrm{E}-3 \mathrm{I}$ \\
\hline \multicolumn{6}{|c|}{ Hormone and light signals } \\
\hline 1612955_at & TC47637 & 4 & Q7IBZ3 & Type-A response regulator & $3,73 \mathrm{E}-1 \mathrm{I}$ \\
\hline 1616694_at & TC46350 & 7 & Q94KS0 & Histidine-containing phosphotransfer protein & I,96E-57 \\
\hline 1614802_at & TC4I8I5 & 7 & Q94KS0 & Histidine-containing phosphotransfer protein & $6,27 \mathrm{E}-59$ \\
\hline 1617513_at & & 8 & Q8LAL2 & IAA26 (Phytochrome-associated protein I) & $6,04 \mathrm{E}-62$ \\
\hline 1618875_s_at & TC46557 & 1 & Q8LAL2 & IAA26 (Phytochrome-associated protein I) & $2,95 \mathrm{E}-63$ \\
\hline 16205/2_at & TC46080 & 8 & Q9ZSY8 & IAA27 (Phytochrome-associated protein 2) & $9,42 \mathrm{E}-86$ \\
\hline \multicolumn{6}{|c|}{ Other light regulated genes } \\
\hline 1614237_a_at & TC38178 & 1 & Q7XAB8 & Light-regulated chloroplast-localized protein & $5,16 \mathrm{E}-23$ \\
\hline 1618107_at & TC46244 & 1 & Q93WJ7 & Leaf Lethal Spot I-like protein & $0,00 \mathrm{E}+00$ \\
\hline 1614720_at & TC46244 & $\mathrm{I}$ & Q93WJ7 & Leaf Lethal Spot I-like protein & $0,00 \mathrm{E}+00$ \\
\hline 1616605_at & TC45785 & 1 & Q8W5A3 & Lethal leaf spot I-like protein & $0,00 \mathrm{E}+00$ \\
\hline 1617173_s_at & & 4 & Q6T619 & ELIP Early light-induced protein & $3,88 \mathrm{E}-54$ \\
\hline 1610360_at & TC38699 & 8 & Q6Q9WI & Ultraviolet-B-repressible protein & $2,47 E-36$ \\
\hline 1620292_at & TC38866 & 8 & Q6Q9WI & Ultraviolet-B-repressible protein & $2,00 \mathrm{E}-33$ \\
\hline \multicolumn{6}{|c|}{ TRANSCRIPTION FACTORS } \\
\hline \multicolumn{6}{|l|}{ MADS-BOX TF } \\
\hline 1621827_at & TC39767 & 1 & Q6UGQ6 & MADS-box protein 15 & $\mathrm{I}, 95 \mathrm{E}-47$ \\
\hline 1621836_at & TC45777 & 7 & Q8LLQ9 & MADS-box protein 5 & $6,25 \mathrm{E}-119$ \\
\hline 1607973_at & TC38620 & 8 & Q9ZTV9 & MADSI-like & $4,87 \mathrm{E}-107$ \\
\hline 1613748_at & TC40277 & 8 & Q8LLR2 & Vitis. MADS-box protein 2, ApI-like, MADS-RIN-like & I,73E-77 \\
\hline \multicolumn{6}{|c|}{ 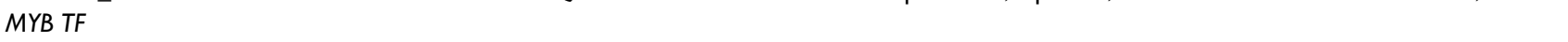 } \\
\hline 1611920_at & TC40303 & 1 & Q9M9A3 & F27JI5.20 (Hypothetical protein) (MYB transcription factor) & $3,83 \mathrm{E}-84$ \\
\hline 1618260_s_at & TC42III & 3 & Q8L5N7 & Myb-related transcription factor VIMYBBI-2, similar to & $4,47 \mathrm{E}-43$ \\
\hline 1620959_s_at & TC48485 & 4 & Q6L973 & Myb-related transcription factor VvMYBAI & $2,04 \mathrm{E}-119$ \\
\hline 16096/2_at & TC47332 & 7 & Q688D6 & Putative myb transcription factor (MYBI6 protein) & $3,|7 E-2|$ \\
\hline 1612686_at & TC48806 & 8 & Q5NDD2 & Putative MYB transcription factor & $6,03 E-84$ \\
\hline 1613486_at & TC45686 & 8 & O04544 & F20P5.26 protein (At Ig70000) (MYB transcription factor) & $\mathrm{I}, 60 \mathrm{E}-74$ \\
\hline 1614932_at & TC48806 & 8 & Q5NDD2 & Putative MYB transcription factor & $6,03 \mathrm{E}-84$ \\
\hline 1618514_at & TC5 I437 & 8 & O22059 & CPC (Putative MYB family transcription factor) & $4,70 \mathrm{E}-\mathrm{II}$ \\
\hline
\end{tabular}


Table I: Selection of genes putatively involved in the regulation of berry ripening. (Continued)

\begin{tabular}{|c|c|c|c|c|c|}
\hline 1619201_at & TC49224 & 8 & Q8GV05 & TRIPTYCHON (MYB transcription factor) & $2,93 \mathrm{E}-29$ \\
\hline \multicolumn{6}{|c|}{ 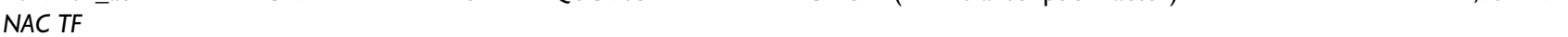 } \\
\hline |62|448_at & TC47I4I & 2 & Q52QRI & NAC domain protein NAC5 & I,39E-27 \\
\hline 1609172 at & TC39120 & 3 & Q52QR2 & NAC domain protein NAC4 & $1,10 E-126$ \\
\hline 1606678_at & TC42489 & 4 & Q8LKN7 & Nam-like protein 17 (Fragment) & $6,53 \mathrm{E}-19$ \\
\hline 1607|20_at & TC46243 & 4 & Q84K00 & NAC-domain containing protein 78 (ANAC078) & I,43E-34 \\
\hline 1621255_at & TC4I700 & 4 & Q8LRL4 & Nam-like protein II & $\mathrm{I}, 85 \mathrm{E}-46$ \\
\hline \multicolumn{6}{|l|}{ WRKY TF } \\
\hline 1609130_at & TC4634I & 3 & Q9FGZ4 & WRKY DNA-binding protein 48 & I,62E-56 \\
\hline 1622333_at & TC40428 & 3 & O22900 & WRKY DNA-binding protein 23 & $5,68 \mathrm{E}-46$ \\
\hline 1611285_s_at & CA809190 & 7 & Q6IEL3 & WRKY transcription factor 68 & I,84E-39 \\
\hline \multicolumn{6}{|l|}{ Homeotic TF } \\
\hline 1616863_at & TC41982 & I & Q8LLD9 & BELI-related homeotic protein 29 (Fragment) & I,20E-78 \\
\hline 1618774_at & TC40997 & I & Q4VPE9 & Lateral organ boundaries-like I (Fragment). & $6,5 \mathrm{IE}-52$ \\
\hline $1611583 \_$at & TC40246 & 3 & Q9FJ90 & Similarity to AP2 domain transcription factor & $2,85 \mathrm{E}-55$ \\
\hline 1609295_at & TC47034 & 3 & P46897 & Homeobox-leucine zipper protein ATHB-7 & $2,4 \mathrm{IE}-53$ \\
\hline 1620170_at & TC42225 & 7 & Q9XHC9 & APETALA2 protein homolog HAP2 & $4,04 \mathrm{E}-46$ \\
\hline 1607|22_at & TC5I295 & 8 & Q546G6 & Homeodomain-leucine zipper protein HAT22 & $4,82 \mathrm{E}-15$ \\
\hline 1607284_at & TC40589 & 8 & Q7Y0Z9 & Bell-like homeodomain protein 3 (Fragment) & $3,12 \mathrm{E}-1 \mid 4$ \\
\hline 161793|_at & TC40I85 & 8 & Q9M276 & Homeobox-leucine zipper protein ATHB-12 & $4,4 \mathrm{IE}-34$ \\
\hline 1615625_at & TC3958I & 8 & 004136 & Homeobox protein knotted-I like 3 (KNAP3) & $6,59 \mathrm{E}-86$ \\
\hline 1613036_at & TC39310 & 4 & Q56R05 & Putative pollen specific LIM domain-containing protein & $2,68 \mathrm{E}-90$ \\
\hline 160656I_at & TC41613 & 7 & 081384 & Putative basic helix-loop-helix DNA binding protein TCP2 & $1,25 \mathrm{E}-20$ \\
\hline 1611070_at & TC49539 & 7 & Q8L8A5 & GRFI-interacting factor I (Hypothetical protein At5g28640) & 7,97E-43 \\
\hline |60659|_at & TC4I789 & 8 & Q8RU28 & Putative SHORT-ROOT (SHR) protein (Fragment) & $1,28 \mathrm{E}-38$ \\
\hline 1610187_a_at & TC47632 & 8 & Q9FL03 & SCARECROW gene regulator & I,IIE-IIO \\
\hline 1619334_at & TC47632 & 8 & Q9FL03 & SCARECROW gene regulator & I,IIE-IIO \\
\hline 1612362_at & TC41970 & 8 & Q6SS00 & YABBY-like transcription factor GRAMINIFOLIA & $3,06 \mathrm{E}-69$ \\
\hline 1613445_at & TC40338 & 8 & Q6XX20 & Mutant cincinnata & $3,64 \mathrm{E}-16$ \\
\hline 161485I_s_at & TC40029 & 8 & Q9SIV3 & Expressed protein (GPRII) (Golden2-like protein I) & $2,10 \mathrm{E}-39$ \\
\hline 1617877_at & TC4I303 & 8 & Q8SBC9 & Transcription factor LIM & I,7IE-92 \\
\hline
\end{tabular}

(a): cluster number as reported in Figure 4.

(b): Uniprot ID, description and E-value of the annotation results obtained by 'blast' and 'GORetriever' analysis.

scription factors of the Ethylene Responsive Factors (ERFs) and AP2/EREBP families. The transcript homologous to tomato LeERF1 and a putative AP2/EREBP transcription factor were induced during berry ripening, while the homologous of ERF3b and another putative AP2/ EREBP transcription factor were repressed. Considering that in tomato, tobacco and Arabidopsis, ERF1 genes are induced by ethylene and wounding and positively regulate downstream genes involved in biotic and abiotic stresses [30], the induced transcription factors that we have identified could be involved in ethylene mediated responses to plant stresses in grape as well $[31,32]$. The Vitis homologues to osmotin, PR-1 and PR-4, downstream genes known to be regulated by ERFs in the species above mentioned, were indeed found to be induced in our study, suggesting that they could be under the same regulatory mechanism. The homologous of tobacco nitrilase NtNIT4B [33], another gene related to the transcriptional control of ethylene responsive defence genes, was found to be induced during ripening in our analysis as well. We could not thus exclude that an additional role of ethylene during grape berry development is related to biotic stress response.
Sequence annotation revealed the modulation of some transcripts homologous to the tomato genes ER6 and ER33, whose functions are still unknown but are reported to be induced by ethylene during tomato ripening [34]. In our experiment, however, these genes did not show strong modulation around véraison and they were all repressed during ripening. On the other hand, a transcript homologous to tomato E8, was found induced in grape, in agreement with tomato and strawberry gene expression data $[35,36]$. Although E8 resembles ACC synthase structure, it apparently exerts a negative control on ethylene biosynthesis [35] and its expression seems affected by the homeotic transcription factor LeMADS-RIN [37]. E8 similar modulation in both climacteric and non-climacteric fruits raises the possibility of a common regulatory mechanism for ethylene homeostasis control in fleshy fruit ripening.

The phytohormone abscisic acid (ABA) regulates processes such as floral transition, embryo maturation, seed development and tolerance to abiotic and biotic stress [38]. In grape berries, ABA begins to accumulate at véraison and is thus considered a good candidate for triggering berry ripening [18]. The present analysis found three ABA- 
related transcripts induced after véraison: one is homologous to the pea nine-cis-epoxycarotenoid dioxygenase 4 and thus putatively involved in ABA synthesis; the other two are homologous to two ABA responsive proteins. Recently, a Vitis dehydrin, DHN1 [39], and some Arabidopsis plasma membrane aquaporins (PIPs) [40], have been reported to be regulated by ABA and involved in stress and senescence, respectively. Interestingly, in our analysis we found VvDHN1 and numerous VvPIP isoforms modulated in a ripening specific way (clusters 4 and 8).

Brassinosteroids accumulate during fruit development and seem to play a key role in determining the onset of ripening in fleshy fruits $[23,41]$. Three Vitis genes involved in brassinosteroid biosynthesis and sensing have been recently cloned and transcriptionally characterized during berry development [23]. Our analysis identified VvBR6OX1, which converts 6-deoxocastasterone to castasterone, the only bioactive brassinosteroid detected in grape [23], and VvBRI1, a brassinosteroid receptor. Their expression profiles were characterized by a peak of induction at TP B, in agreement with previous data [23].

\section{Light stimulus}

We identified numerous transcripts putatively involved in the circadian rhythm oscillatory system. In particular, one member of the His-to-Asp two-component signal transduction family, homologous to Arabidopsis APRR2, and one transcription factor of the Constans-like family, showed a decreasing profile after véraison, while a kinase homologous to WNK1 [42], and two transcription factors, belonging to the Constans-like and Early flowering family respectively [43], displayed a positive regulation, more marked after véraison. A transcript homologous to Flowering locus $\mathrm{T}$, putatively coordinating the circadian rhythm with the flowering switch [44], appeared expressed until TP B, thus suggesting its involvement in signalling circuits during early berry development.

Some transcripts putatively involved in light and hormonal signalling cross-talk were also identified. IAA26/phytochrome associated protein 1 and IAA27/phytochrome associated protein 2, involved in light and auxin signal transduction, were down regulated after véraison. Members of the His-to-Asp family, such as Type A response regulators, pseudo response regulators and histidinecontaining phosphotransfer proteins, are known to mediate the responses to light, osmotic, cytokinin and ethylene stimuli (reviewed in [45]) and were found modulated during the process of berry development.

\section{Transcription factors}

In our study, we identified 125 transcription factors, among which members of the myb, MADS-box, NAC and
WRKY families and some homeotic and developmentspecific genes.

Within the myb family, we found two previously isolated Vitis genes: VvmybA1, which showed a ripening-specific profile highly correlated to those of UDP-glucose:flavonoid 3-O-glucosyltransferase, glutathione-S-transferase 4 and the caffeoyl-CoA O-methyltransferase as described in [46], and a VlmybB-like gene, which was induced during berry development as reported for Vitis labruscana [47]. The recently characterized grape Vvmyb5A, proposed to be involved in the regulation of the phenylpropanoid pathway [48], was found to be expressed invariantly at low levels in all years during this study interval (1621471_s_at), thus not supporting a ripening-specific function for this gene.

Concerning the MADS-box family of transcription factors, only one previously characterized grape gene was present in the regulated set, namely VvMADS5, which was highly induced during early berry development, in agreement with [49]. Both VvMADS1 (1619742_at) and VvMADS4 (1614965_at), which were previously reported as abundant transcription factors involved in berry development $[49,50]$, were highly expressed at all time-points in our experiment and thus not included in the regulated set.

1613748_at could represent the homologue of tomato LeMADS-rin $(\mathrm{E}$-value $=8.2 \mathrm{e}-50,68 \%$ identity), which is part of the developmental signalling system that initiates ripening in this fruit [51]. However, while LeMADS-rin is induced from breaker stage to ripeness, its putative grape homologue was slightly induced until véraison and then showed a decreasing profile. This behaviour could either suggest that the grape transcript is not the homologue of LeMADS-rin or that its role is different in non-climacteric fruits.

Five transcripts with homology to NAC transcription factors appeared modulated, all in a positive way in the study interval. This is of interest as recent transcriptome studies report the regulation of NAC transcription factors family not only during biotic and abiotic stress responses, but also in many other processes, such as fruit development and ABA signalling (reviewed in [52]).

Three transcripts homologous to the Arabidopsis WRKY family were also modulated. In plants, the WRKY family is quite large (74 members in Arabidopsis and 90 in rice) and its members participate in numerous cellular processes, such as defence and hormonal signalling, developmental programs and fruit maturation (reviewed in [53]).

Nine putative homeotic genes previously identified in floral development studies and 11 transcripts involved in 
other morphological and/or developmental processes were modulated in our study although their connection to fruit development has not yet been reported and will need further investigation. 1606591_at and 1619334_at correspond to two transcriptional regulators, SHORT ROOT and SCARECROW, which in Arabidopsis are both implicated in developmental root layers specification [54]. As they were co-expressed in our analysis, they might interact in berry as well.

Though not present in the regulated gene set, 1616455_s_at, corresponding to the grape VvMSA transcription factor ought to be mentioned. VvMSA is induced by sugar and ABA and positively regulates VvHT1 expression $[55,56]$. In our study, 1616455_s_at was very highly expressed at all three time-points, in agreement with previous proteomic and gene expression studies $[7,55,57]$.

\section{Genes responsible for berry phenotypic traits and stress response}

Berry development involves major transcriptional changes of genes directly responsible for the biochemical and phenotypical characteristics of each growth stage. Several protein families linked to these phenotypic traits have been expansively described in past studies focussed on single or small gene sets [58] and recently in a genomewide study [11]. The present work provides an extensive description of gene expression during ripening, reporting for the first time the occurrence of an oxidative burst immediately after véraison. The complete list of the genes discussed in the text is in part B of Additional file 7, ordered by GO functional category; for clarity, they are further divided according to more specific GO metabolisms, when possible.

\section{Cell wall metabolism}

The previously described involvement of specific gene families in berry growth and softening [59] was confirmed in the present study. Among these, there were pectin modifying enzymes (methylesterase, invertase/pectin methylesterase inhibitor, pectate lyase, pectinesterase), extensins and expansins, grape ripening-induced proteins (GRIP 3, $13,15,22$ and 28) and xyloglucan endotransglycosylases (XETs) $[8,59]$. In particular, 10 members of the XET family were modulated and four of these very strongly during ripening (1613415_at, 1615809_at, 1619355_at, 1621251_s_at, cluster 4). These induced isoforms are homologous to the tomato SIXTH5, 6 and 8 XET isoforms, recently defined as Group 3 XETs [60]. Although from the analysis of the tomato fruit cDNA libraries only SIXTH5 was found expressed [60], our array-based gene expression analysis suggests that all the three isoforms of group 3 could be involved in fruit development sharing a common function. Vitis XET isoforms have been proposed to be implied in brassinosteroids mediated berry cell wall softening [23].

\section{Stress response}

The present analysis identified 270 transcripts related to plant stress perception and response which fall into the "response to stimulus" GO category.

Sixty-nine transcripts were involved in plant biotic stress response, and they were mostly accumulating during ripening. The induction of defence genes including GRIPs (GRIP 22, 28, 31, 32, 51, 61), thaumatins, lipid transfer proteins, chitinases, $\gamma$-thionins and pathogenesis related proteins during berry ripening, confirmed previous results [8].

A particular kind of stress response which has been reported during fruit development in several species such as tomato [61], strawberry [20], avocado [62], pineapple [63] and pear [64], is the response to oxidative stress. In recent years a dual role for reactive oxygen species (ROS) in plants has been identified: toxic by-products of the aerobic metabolism and important regulators of growth, development and defence reactions. A complex network of genes finely tuning ROS concentration as the result of their production and scavenging has been documented [65].

The occurrence of oxidative stress during grape berry development has been rather controversial: catalase was identified among the abundant proteins in the ripe berry [57], but at the transcriptional level the typical oxidative stress markers seemed absent or negatively regulated [10]. The present study clearly indicated that oxidative processes take place also during berry ripening and that an enzyme-mediated scavenging system is activated. Berry $\mathrm{H}_{2} \mathrm{O}_{2}$ content increased significantly in correspondence of the véraison stage, reaching its maximum one-two weeks after, and then decreasing at a slower pace towards harvest time (Fig. 6). At least 32 transcripts of the modulated core set appeared involved in the enzymatic detoxification from $\mathrm{H}_{2} \mathrm{O}_{2}$ and the other ROS species which accumulate in the cell starting from véraison (Fig. 7). Some of them, such as ascorbate peroxidase, glutathione peroxidase and peroxiredoxins, catalyze the direct reduction of hydrogen peroxide to water; the others (thioredoxins, glutaredoxins, glutathione-S-transferases and metallothioneins) regulate the balance of the oxidized and reduced forms of the antioxidants ascorbic acid and glutathione. Although the regulation of the detoxifying enzymes must be very complex, isozyme specific, and occurring at different levels (transcriptional- and post-transcriptional regulation, subcellular compartimentalization, etc) as previously shown for some of them [61], most of the enzymes shown in Figure 7 appeared to be unchanged from TP A to TP B and be 


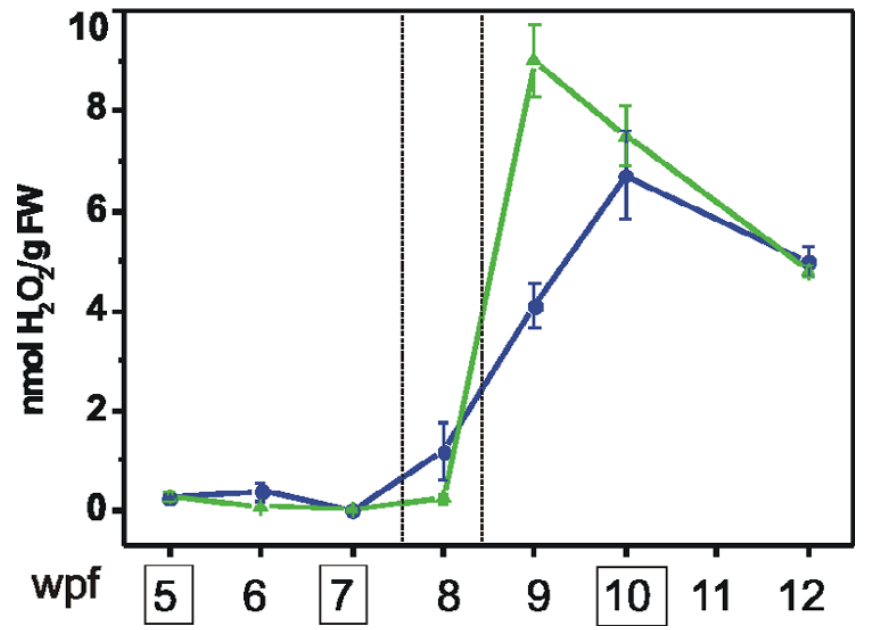

Figure 6

$\mathrm{H}_{2} \mathrm{O}_{2}$ content during berry ripening in 2005 (blue) and 2006 (green) samples. Data are means \pm SE of three replicates. The weeks post flowering (wpf) corresponding to the samples used for the hybridization experiments are enclosed in boxes. Véraison time is delimited within dotted lines.

strongly modulated from TP B to TP C. Further studies are needed to address how ROS molecules are taking part to these regulatory circuits and what is the main reason of ROS accumulation at véraison. The superoxide dismutase (SOD) and catalase (CAT) transcripts which also codify for two important detoxifying enzymes were not significantly modulated in our study. The CAT gene (1618705_s_at) was highly expressed in all the three TPs, the SOD encoding transcripts (5 probes) showed very different expression levels but no one fell into the modulated core set.

The Nudix gene family may also be involved in the oxidative stress response, as three transcripts were found modulated (one in cluster 3 and two in cluster 8). In Arabidopsis, Nudix hydrolases appear to be involved in the sanitization of the oxidized nucleotide pool accumulating during oxidative stress [66].

Ten genes involved in programmed cell death (PCD) appeared to be modulated. In particular, three transcripts with putative function of PCD suppression are grouped in cluster 1 , while a putative death associated protein is induced after véraison (cluster 4). In addition, listed in the protein metabolism category there are two cysteine proteases (cluster 4) and a cystatin-like gene (cluster 5). Our data are insufficient to draw any conclusion, however they may suggest the occurrence of a PCD process in grape berry during ripening, as highlighted for strawberry [20].

\section{Sugar/acid ratio and water balance}

Our results support the model of sugar phloem unloading via an apoplastic pathway by means of plasma membrane sucrose and hexoses transporters as proposed by [67] and recently confirmed by $[68,69]$. Four sugar transporters were found modulated: the hexose transporter VvHT6 (1615257_at and 1619691_at) was positively modulated during ripening (cluster 3 ), as previously described [10]; interestingly, a transcript homologous to a sugar transporter isolated in Citrus fruit was also induced while the forth transcript, which is homologous to a sugar transporter from tomato root is repressed. The sucrose transporter VvSUC27 appeared dramatically down-regulated after véraison, as already reported in the cv. Syrah [70], while, VvSUC11 and VvSUC12, transporters with a ripening specific expression profile [70], were highly expressed at all time points in our experiment.

There is evidence that during berry ripening, sucrose is hydrolysed in the cell wall and then re-synthesised in the cytoplasm following hexose uptake [69]. Our data corroborate this mechanism as three sucrose-phosphate synthases (cluster 3 and 4), a sucrose synthase (cluster 4), a sucrose-6-phosphate phosphatase (cluster 4), a UDPsugar pyrophosphorylase (cluster 2) and a beta-phosphoglucomutase (cluster 4) were all up-regulated during ripening. Vacuolar invertases, VvGIN1 and VvGIN2, both resulted highly expressed until véraison (cluster 8 ), as previously described $[57,71]$. The existence of 'futile cycles' of sucrose synthesis and breakdown has been demonstrated in other fruits, such as tomato, where the relative rate of synthesis and breakdown cycles controls the rate of sugar and starch accumulation [72].

The early expression of a phosphoenolpyruvate carboxylase isoforms (cluster 8) well correlated with the rate of malate accumulation during the first phase of berry development. Furthermore, a vacuolar pyrophosphatase (1614834_at) putatively involved in malate vacuolar uptake was expressed early and then induced during ripening (cluster 5), as previously observed [73]. At véraison, when malate decompartimentalization and breakdown take place [67], induction of malate degrading enzymes, such as malic enzyme and phosphoenolpyruvate carboxykinase (cluster 1) and mitochondrial malate dehydrogenase (cluster 8) was observed. The profiles observed for grape alcohol dehydrogenase 2 and a putative short chain alcohol dehydrogenase (cluster 4 and 5, respectively) and three isoforms of aldehyde dehydrogenase (one in cluster 4 and two in cluster 8) may be indicative of a shift to an aerobic fermentative metabolism during ripening [74]. The alpha subunit of the pyruvate dehydrogenase complex was induced after véraison (cluster 5); however, this enzyme is known to be highly regulated post-translationally, allosterically by NADH and covalently by reversible 


\begin{tabular}{|c|c|c|c|c|c|}
\hline Protein Annotation & Affy ID & Cluster & $\mathbf{B} / \mathbf{A}$ & C/B & \multirow{5}{*}{$\begin{array}{l}>2 \\
>1.5 \\
>1.0 \\
>0.5\end{array}$} \\
\hline \multicolumn{5}{|c|}{ Ascorbate peroxidase: $2 \mathrm{Asc}+\mathrm{H}_{2} \mathrm{O}_{2}>2 \mathrm{MDA}+2 \mathrm{H}_{2} \mathrm{O}$} & \\
\hline Cytosolic ascorbate peroxidase 2 & 1618137_at & 1 & & & \\
\hline L-ascorbate peroxidase, chloroplast precursor & 1618209_at & 8 & & & \\
\hline \multicolumn{5}{|l|}{ Alternative oxid as e: $2 \mathrm{e}+2 \mathrm{H}^{+}+\mathrm{O}_{2}>\mathrm{H}_{2} \mathrm{O}$} & \\
\hline Alternative oxidase $1 \mathrm{~b}$ & 1607193_at & 4 & & & $<-0.5$ \\
\hline Alternative oxidase $1 \mathrm{a}$ & 1615827_at & 4 & & & \\
\hline \multicolumn{5}{|l|}{ Peroxidase: $2-\mathrm{SH}+\mathrm{H}_{2} \mathrm{O}_{2}>\mathrm{S}-\mathrm{S}+2 \mathrm{H}_{2} \mathrm{O}$} & $<-2.0$ \\
\hline Peroxidase 17 precursor & 1614361_at & 1 & & & \\
\hline Peroxidase precursor & 1621431_at & 5 & & & \\
\hline Peroxidase precursor & 1608586_at & 8 & & & \\
\hline Secretory peroxidase & 1613132_s_at & 8 & & & \\
\hline Thioredoxin peroxidase & 1607497_at & 7 & & & \\
\hline Glutathione peroxidase & 1609654_at & 4 & & & \\
\hline \multicolumn{5}{|l|}{ Peroxiredoxins: $2 \mathrm{P}-\mathrm{SH}+\mathrm{H}_{2} \mathrm{O}_{2}>\mathrm{P}-\mathrm{S}-\mathrm{S}-\mathrm{P}+2 \mathrm{H}_{2} \mathrm{O}$} & \\
\hline Peroxiredoxin & 1607335_at & 5 & & & \\
\hline Peroxiredoxin $\mathrm{Q}$ & 1614204_at & 8 & & & \\
\hline \multicolumn{5}{|l|}{ Thioredoxins: P-S-S-P $+2 \mathrm{H}>2 \mathrm{P}-\mathrm{SH}$} & \\
\hline Putative thioredoxin $\mathrm{F}$ & 1617646_at & 1 & & & \\
\hline Putative thioredoxin $\mathrm{m} 2$ & 1614231_s_at & 8 & & & \\
\hline Thioredoxin M-type, chloroplast precursor & 1615926_at & 8 & & & \\
\hline \multicolumn{5}{|l|}{ Glutaredoxins: $\mathrm{DHA}+2 \mathrm{GSH}>\mathrm{Asc}+\mathrm{GSSG}$} & \\
\hline Glutaredoxin-like & 1611572_at & 3 & & & \\
\hline Putative glutaredoxin & 1614477_s_at & 8 & & & \\
\hline \multicolumn{5}{|c|}{ Glutathione S-tran sferase: $\mathrm{R}-\mathrm{O}-\mathrm{OH}+2 \mathrm{GSH}>\mathrm{R}-\mathrm{OH}+\mathrm{GSSG}$} & \\
\hline Glutathione S-transferase, Phi class* & 1619917_s_at & 4 & & & \\
\hline Glutathione S-transferase, Phi class* & 1614658_a_at & 4 & & & \\
\hline Glutathione S-transferase, Phi class* & 1615117_a_at & 4 & & & \\
\hline Glutathione S-transferase, Thau class & 1612535_s_at & 4 & & & \\
\hline Glutathione S-transferase (Fragment), Phi class & 1606607_at & 4 & & & \\
\hline Glutathione S-transferase GST 18, Thau class & 1608260_at & 4 & & & \\
\hline Glutathione S-transferase GST 14, Thau class & 1609202_at & 1 & & & \\
\hline Glutathione S-transferase, Thau class & 1607482_at & 8 & & & \\
\hline Glutathione S-transferase, Thau class & 1606595_at & 1 & & & \\
\hline Glutathione S-transferase, Thau class & 1621395_at & 1 & & & \\
\hline 2,4-D inducible GST, Thau class & 1613204_at & 2 & & & \\
\hline \multicolumn{5}{|l|}{ Metallothioneins: $\mathrm{DHA}+2 \mathrm{GSH}>\mathrm{Asc}+\mathrm{GSSG}$} & \\
\hline Metallothionein $2 b$ & 1615445_at & 8 & & & \\
\hline Metallothionein-like protein & 1613521_at & 7 & & & \\
\hline Metallothionein-like protein & 1607049_s_at & 7 & & & \\
\hline Putative metallothionein-like protein (GRIP 24) & 1610539_x_at & 1 & & & \\
\hline
\end{tabular}

\section{Figure 7}

Genes involved in the response to oxidative stress modulated during ripening. List of the ROS-scavenging genes found in the modulated core set and comparative analysis of their expression in the three sampling TPs. Log 2 ratios of the expression values observed in TPs $A, B$ and $C(B / A$ and $C / B)$ are visualized by a colour scale, where red indicates an increase and green a decrease. For each functional group the corresponding enzymatic reaction is reported. * refer to probesets probably corresponding to the same transcript.

phosphorylation. Our analysis identified two mitochondrial pyruvate dehydrogenase kinase isoforms, one of which induced in a ripening-specific way. Two enzymes of the glycolysis/gluconeogenesis pathways, such as enolase and glyceraldehyde-3-phosphate dehydrogenase (cluster
5 and 4, respectively) were induced during ripening, suggesting their involvement into the acid/sugar interconversion and/or the fermentative metabolism. 
About $90 \%$ of ripe berries fresh weight is comprised of water whose transport into the berry vacuole is mediated by aquaporins. In our study we detected 13 transcripts coding for aquaporin isoforms: eight belong to the plasma membrane intrinsic protein family, four to the tonoplast intrinsic protein family and one to the NOD26like intrinsic protein family. All isoforms were highly expressed in the pre-véraison phase and three of them, coding for the putative aquaporin PIP2-1, were also induced during ripening. A similar modulation has been recently observed in Citrus, although the induced isoforms during fruit development, belong to the TIP family [16].

\section{Secondary metabolism}

The "secondary metabolism" GO functional category included genes involved in the synthesis of aromatic and volatile compounds, such as terpenes and benzenoid compounds; antioxidant compounds, such as polyphenols and vitamin $\mathrm{E}$, and genes of the phenylpropanoid pathway. Due to its importance for berry quality traits, we restricted our analysis to the latter pathway. Most structural genes of this branched pathway, which starts with phenylalanine ammonia lyase, were present among the regulated genes of the current study. During ripening, the genes coding for the enzymes acting in flavonols synthesis (flavonol synthase, cluster 3), stilbenes synthesis (three isoforms of stilbene synthase, cluster 4) and anthocyanins synthesis (UDP-glucose:flavonoid 3-O-glucosyltransferase, cluster 4) were induced, while those involved in tannins synthesis (anthocyanidin reductase and leucoanthocyanidin reductase) were repressed. The profiles of genes coding for phenylalanine ammonia lyase, chalcone isomerase 1 and flavonoid 3'5'-hydroxylase showed a decrease until véraison followed by an induction during ripening, confirming previous data [75]. Two isoforms of 4-coumarate-CoA ligase with opposite profiles were detected (clusters 3 and 8) and their pattern of expression is consistent with an involvement into the anthocyanin and lignin synthesis pathways, respectively.

Eleven members of the ATP-binding cassette $(\mathrm{ABC})$ and multidrug and toxic compound extrusion (MATE) transporters families which are involved in several cellular transport processes (reviewed in [76]), were modulated. Among them, the three transporters positively modulated during berry development (1610275_at, 1607763_at, 1616929_at) represent possible candidates for secondary metabolites transport.

\section{Gene expression profiles highly influenced by seasonal variation}

The influence on gene expression by environmental conditions is well reported in literature [77] and was evident in our PCA analysis (Fig. 2). The comparison of the three sampling years highlighted a large number of genes modulated only during one or two seasons, and thus excluded by the core set of ripening-specific genes: 938 genes in 2003, 2530 in 2005 and 2143 in 2006. Two examples are the isoform 2 of the phenylpropanoid biosynthetic genes chalcone synthase and chalcone isomerase whose expression profiles are depicted in Fig. 8E and 8F; such discrepancy in their modulation is indeed expected for nonripening specific isoforms. Although these genes are probably highly affected by changes in the environment their GO functional categories distribution was not significantly different to that of the core set genes (data not shown). This result suggests that, on a long time frame, the plant reacts to the seasonal variations by adjusting the whole metabolism, and not just a part of it, to maintain homeostasis.

The group of genes modulated in all three seasons with different transcriptional profiles, deserves in our opinion a special discussion as they are likely involved into the ripening process but in the meantime highly influenced by the climatic conditions. These 223 genes represent about $13 \%$ of the modulated gene set (1700 transcripts). Among them, at least four transcripts are putatively involved in light perception and signalling: cryptochrome 1 (1615199_at) and pseudo-response regulator 9 (APRR9, 1616872_at) showed opposite behaviours in 2003 vs. 2005 and 2006 (Fig. 8A and 8B); pseudo-response-regulator 7 (APRR7, 1608006_at) displayed a "V" profile in 2003 and 2005 and the opposite pattern during 2006; finally LONG HYPOCOTYL 5 (HY5, 1609930_at) showed a different profile from TP A to TP B in 2003 and 2005 vs. 2006 while it was repressed in all three seasons from TP B to TP C. Interestingly, APRR7 and APRR9 seem involved in the temperature responsiveness of the Arabidopsis circadian clock, synchronizing photo- and thermo-cycles [78] and HY5 is a positive regulator of light signalling which acts downstream of several photoreceptors and mediates the response to different types of light (red/far red, blue and UV-B $[79,80])$.

Out of the three previously characterized grape hexose transporters present on the chip, two (Vvht1 and Vvht2) seemed influenced by the season. Vvht1 (1616083_at) showed a "V" profile in all the three years, but with different slopes, suggesting a strong environmental conditioning along with the reported cultivar specificity $[10,81,82]$. Unlike Vvht1, Vvht2 is considered a ripening specific isoform $[10,82,83]$; however, the present work highlighted a certain degree of variability in its induction time in the three years (1614764_at, Fig. 8C). A similar behaviour was observed for another ripening specific gene, namely the isoform 3 of the chalcone synthase family $[75,84]$ (Fig. 8D). 

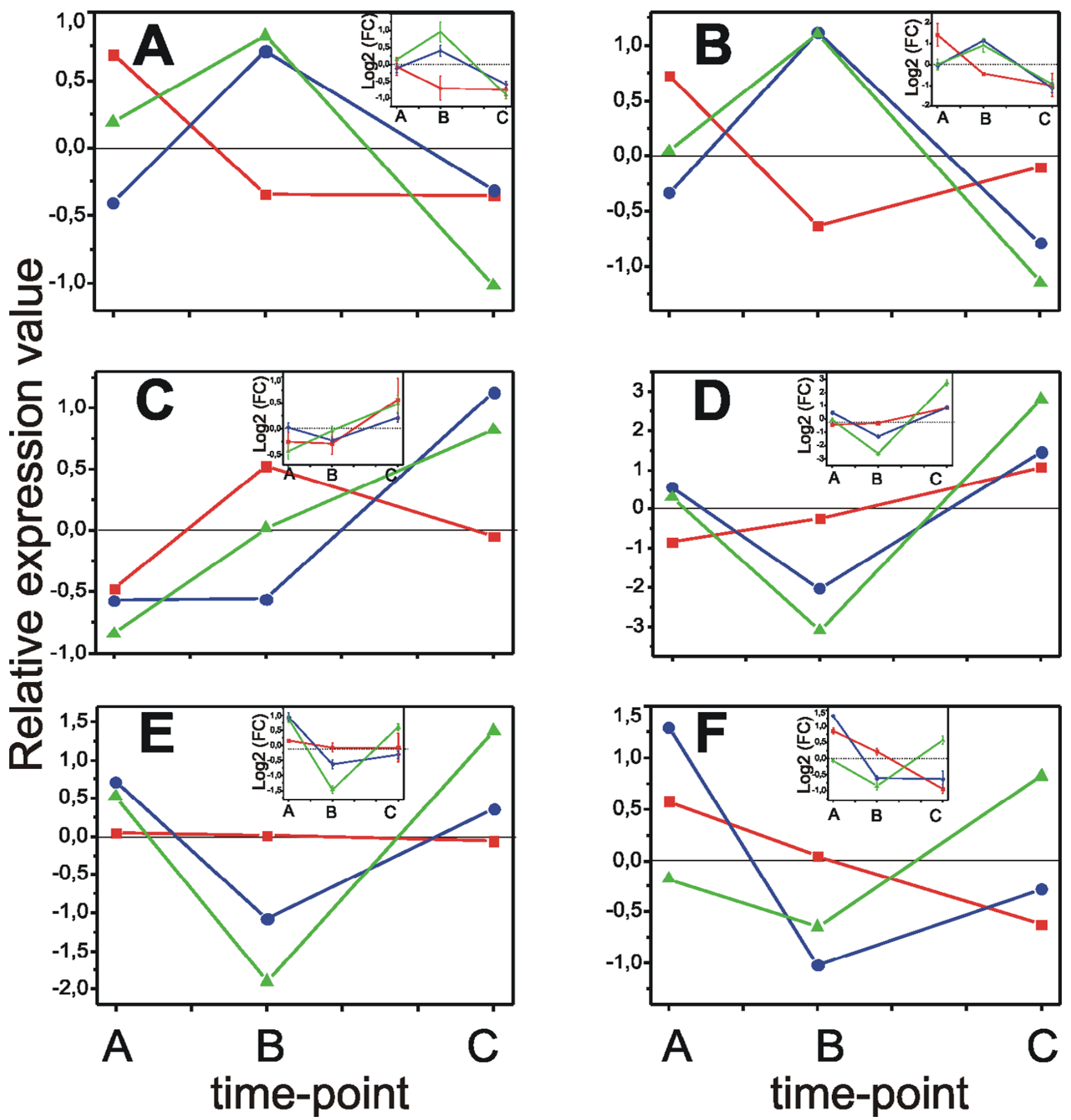

Figure 8

Real time RT-PCR validation of the expression profiles of six genes highly influenced by the season. A:

1615199_at, Cryptochrome I; B: 1616872_at, Pseudo response regulator 9; C: 1614764_at, VvHT2, hexose transporter 2; D: I606663_at, VvChs3, chalcone synthase isoform 3; E: 1607732_at, VvChs2: chalcone synthase isoform 2; F: I620424_at, VvChi2, chalcone isomerase isofom 2. 2003 profiles are represented in red, 2005 in blue and 2006 in green. Relative expres-

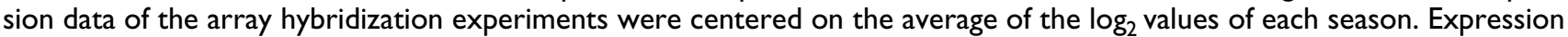
profiles measured by RT-PCR experiments (insets) were first centered on the mean $\mathrm{C} t$ value calculated on the three seasons for each gene and then $\log _{2}$ transformed. RT-PCR data are reported as means \pm SE of three technical replicates. 
To ensure that the observed differences among seasons and among gene isoforms were not technical artefacts, the expression profile of six genes was validated by real time RT-PCR (Fig. 8, insets). In all but one case (Vvht2 year 2003) there was complete agreement with the array data.

\section{Conclusion}

The time-course gene expression analysis of Pinot Noir berry development, based on a systematic and genomewide approach, has identified the occurrence of two well distinct phases along the process. The pre-véraison phase represents a reprogramming stage of the cellular metabolism, characterized by the expression of numerous genes involved in hormonal signalling and transcriptional regulation. The post-véraison phase is characterized by the onset of a ripening-specialized metabolism responsible for the phenotypic traits of the ripe berry. A similar switch in fruit metabolism has been recently reported also in Citrus [16]. Five functional categories are overrepresented in this ripening-specialized metabolism: cell wall, carbohydrate and secondary metabolisms, stress response and photosynthesis; the latter being obviously switched off. An oxidative burst, previously not detected in grapevine and characterized by a rapid accumulation of $\mathrm{H}_{2} \mathrm{O}_{2}$ starting from véraison, was demonstrated. A concurrent modulation of the enzymatic ROS scavenging network was also highlighted, suggesting a possible role of ROS species in the regulation of berry ripening. We have found a very large number of transcription factors and transcripts related to hormonal metabolism and signal transduction that appear to be regulated during berry development. These represent $19 \%$ of the modulated gene set, corroborating the widely-accepted hypothesis that fruit development is under tight transcriptional control. These data provide the basis for subsequent correlation analysis aimed at highlighting gene co-regulation and metabolic networks [85]. Our strategy to perform the experiment during three seasons allowed us to restrict the dataset of modulated genes to those more likely to be ripening-specific. Nonetheless, we were able to separate ripening specific isoforms within gene families and to identify ripening related genes which appeared strongly regulated also by the seasonal weather conditions.

In perspective, the wealth of information we present here will provide a new extended platform to study the complex process of grapevine berry development and more in general of non-climacteric fruits.

\section{Methods}

\section{Plant Material and Experimental Design}

Ten grape clusters of Vitis vinifera cv. Pinot Noir were collected weekly between 8 a.m and 9 a.m, from flowering to over-ripening during 2003, 2005 and 2006 seasons at the IASMA study site 'ai Molini' (San Michele all'Adige-TN
Italy). To be more representative of the canopy five clusters were collected from the north side (shady) and the other five from the south side (sunny). Berries were rapidly detached with their petioles and divided randomly into groups of 50 that were either immediately frozen for subsequent transcriptional analysis or subjected to biochemical analyses, including must acid and sugar content, average berry weight determination and anthocyanin concentration [68]. Based on these parameters, the peak of acidity just before véraison was estimated and two additional time-points (two weeks before and three weeks after véraison) were chosen for gene expression analysis. The three TPs correspond to the 33, 34 and 36 of the modified E-L system [12]. For each time-point three biological replicates were obtained by performing an independent RNA extraction from three sub-pools of 8 berries derived from the group of 50 berries. Consequently nine arrays were utilized for each season.

\section{RNA Preparation, Array Hybridization, Data Analysis}

Total RNA was extracted from frozen deseeded berries according to [86] and further purified using RNeasy spin columns (Qiagen). RNA quality and quantity were assessed by gel-electrophoresis and by absorbance measurements. Ten $\mu \mathrm{g}$ of each sample were sent to the IFOM (Milan-I) Affymetrix platform facility for probe synthesis, GeneChip ${ }^{\circledast}$ Vitis arrays hybridization, washing, staining and scanning with GeneChip Scanner 3000, according to the 'Affymetrix GeneChip ${ }^{\circledast}$ Expression Analysis Technical Manual'. Data referred to 2003, 2005 and 2006 were analyzed independently in order to obtain one set of differentially expressed genes for each season. Bioconductor packages [87] in R v.2.1.0 [88] were used for the statistical analyses. Data were pre-processed using the GCRMA method [89], which performs background correction, quantile normalization and summarization, and returns $\log 2$ converted expression values. Biological replicates quality was assessed by means of R-squared coefficient, which represents the fraction of variance explained by a linear model. For variation assessment of the gene expression values between all 27 data sets, data from the three seasons were scaled and Principal Components Analysis (PCA) was performed. In order to identify genes modulated during berry development, a filtering step based on the inter-quantile range method $(\mathrm{IQR}=0.25)$ was used. This restricted dataset, enriched in differentially expressed genes, was suited to run a multiclass comparison method of Significance Analysis of Microarrays (SAM), with false discovery rate $=0.15 \%$ [13]. SAM output was further restricted to genes with fold-change greater than 2 in at least one of the two comparisons (TP A vs. TP B and TP B vs. TP C). The final datasets relative to the three seasons were intersected obtaining a dataset of 1700 common modulated genes. Pearson's correlation coefficients between genes profiles relative each pair of seasons were 
then calculated and a threshold of 0.5 was fixed to obtain a berry ripening-modulated core dataset of 1477 genes. Expression profiles clustering was carried out by the kmeans method $(k=12)$ with Pearson's correlation distance using the T-MeV software [90]. The number of clusters was manually reduced to eight, which represents the minimum number of expression profiles considering three time-points.

\section{Affymetrix Vitis Array}

The GeneChip ${ }^{\circledR}$ Vitis vinifera genome array (Affymetrix) consists of 16,436 probesets: 14,496 derived from $V$. vinifera transcripts and 1,940 derived from other Vitis species or hybrids transcripts. It can interrogate 12,908 GenBank accessions of $V$. vinifera and 1,547 of other Vitis species or hybrids. Sequences used in the design of the Vitis GeneChip ${ }^{\varpi}$ were selected from GenBank, dbEST, and RefSeq. The sequence clusters were created from the UniGene database (Build 7, Oct 2003). V. vinifera sequences represented on the chip correspond to 10,042 TIGR Tentative Consensus and 1,940 Singletons (Release 4, Sept 2004), while 102 GenBank accessions are not present in the TIGR database. Overall chip redundancy is estimated to be $16.6 \%$.

\section{Annotation}

The consensus sequence of each Vitis GeneChip ${ }^{\varpi}$ probeset (available at [91] was analyzed to identify the coding sequence (cds) by means of an in-house developed method, which combines the results of two cds predictors: Estscan [92] and FrameFinder (at ESTate, [93]). 13,923 cds were obtainded and used to interrogate the Uniprot database (Sept. '05) by means of 'blastp', while the remaining 2,513 sequences were used to interrogate the same database by means of 'blastx' [94]. Blast results (Evalue <e-10) with GO associated terms ([14]) were analyzed by the in-house developed program 'GORetriever' for annotation and 12,242 sequences were annotated with high confidence $(>80 \%$ ). The automatic annotation results for the 1700 modulated genes were manually inspected and integrated with GO 'biological process' terms supported by literature evidences.

Genes were grouped into 17 functional categories based on GO 'biological process' terms by means of the in-house developed program 'GOSlimer' [see Additional file 4 for the list of the GO categories and Additional file 3 for genes annotation]. Functional categories distribution in the modulated and chip sequences were compared by means of Chi square and Fisher statistical tests (p-value $<0.001)$.

\section{Determination of the $\mathrm{H}_{2} \mathrm{O}_{2}$ content}

$\mathrm{H}_{2} \mathrm{O}_{2}$ content was measured by a fluorimetric assay based on the substrate 10-acetyl-3,7-dihydroxyphenoxazine which is oxidized in the presence of $\mathrm{H}_{2} \mathrm{O}_{2}$ and peroxidase, using the Amplex Red Hydrogen Peroxide/Peroxidase Assay (Molecular Probes, Eugene, USA) following the manufacturer's instructions. Ten frozen berries for each time point considered were ground in liquid nitrogen and $0.2 \mathrm{~g}$ of fine powder were then solubilized, for each replicate, with $0.5 \mathrm{ml}$ of $50 \mathrm{mM}$ phosphate buffer ( $\mathrm{pH} 7.4$ ) and kept $5 \mathrm{~min}$ on ice. After centrifugation at 20,000 g for 15 min, the cleared supernatant was extracted with an equal volume of 2:1 (v/v) chloroform:methanol mixture and centrifuged at $12,000 \mathrm{~g}$ for $5 \mathrm{~min}$. To perform the $\mathrm{H}_{2} \mathrm{O}_{2}$ assay $50 \mu \mathrm{l}$ of the aqueous phase were added to $50 \mu \mathrm{l}$ of the reagent working solution. After $30 \mathrm{~min}$ of incubation at $25^{\circ} \mathrm{C}$, relative fluorescence (excitation at $544 \mathrm{~nm}$ and emission at $590 \mathrm{~nm}$ ) was measured in a black 96 wells plate. Absolute quantification was determined by the use of a $\mathrm{H}_{2} \mathrm{O}_{2}$ standard curve.

\section{Real time RT-PCR}

First strand cDNA synthesis was performed on one mRNA sample of the three biological replicates used for the microarray experiments using the SuperScript ${ }^{\mathrm{TM}}$ III Reverse Transcriptase kit (Invitrogen) according to the manufacturer instructions. Primers [see Additional file 8 for sequences] and CDNA were mixed with the Platinum ${ }^{\circledast}$ SYBR $^{\circledast}$ Green qPCR SuperMix-UDG (Invitrogen) and the reaction was carried out on an ABI PRISM 7000 Sequence Detection System (Applied Biosystems). Cycling conditions were: $50^{\circ} \mathrm{C}$ for 2 minutes, $95^{\circ} \mathrm{C}$ for 2 minutes, then 40 cycles of $95^{\circ} \mathrm{C}$ for 15 seconds and $60^{\circ} \mathrm{C}$ for 1 minute. Raw data were analyzed with ABI PRISM 7000 SDS software to extract $\mathrm{Ct}$ values and with the LinReg software to calculate the reaction efficiency [95]. Relative expression of each gene (target) was then calculated according to the equation by [96] using actin for normalization (reference) and centered on the mean Ct calculated on the three seasons and on the three time points (control): RelExp $=\mathrm{E}_{\text {(tar- }}$ get) $\exp \left[\Delta \mathrm{Ct}_{\text {(target) }}\right.$ (sample-control) $] /$

$\mathrm{E}_{(\text {reference) }} \exp \left[\Delta \mathrm{Ct}_{\text {(reference) }}\right.$ (sample-control) $]$.

\section{Data Availability}

All microarray expression data produced by this work are available at EBI ArrayExpress [97] under the series entry EMEXP-1282.

\section{Abbreviations \\ cDNA Complementary DNA}

EST Expressed Sequence Tag

GO Gene Ontology

NCBI National Center for Biotechnology Information

SAM Significance Analysis of Microarrays 
TIGR The Institute for Genomic Research

TAIR The Arabidopsis Information Resource

\section{Authors' contributions}

SP has made substantial contribution to conception, data analysis and manuscript drafting. MP participated in the GO annotation of the sequences, performed profiles clustering and revised critically the manuscript. AM has contributed to data analysis and revised critically the manuscript. AC, LD and PF contributed to sequence annotation. ADR carried out samples collection, RNA isolation, the biochemical assays and the real time PCR experiments. RV contributed to critically revise the manuscript. RV participated to project's design and coordination. CM has made substantial contribution to conception, project coordination and helped in drafting the manuscript. All authors read and approved the final manuscript.

\section{Additional material}

\section{Additional file 1}

Winkler index. Winkler index of the study site calculated for the seasons 2002-2006

Click here for file

[http://www.biomedcentral.com/content/supplementary/14712164-8-428-S1.tiff]

\section{Additional file 2}

Data quality assessment. R-squared coefficient analysis on the 27 hybridization data sets.

Click here for file

[http://www.biomedcentral.com/content/supplementary/1471-

2164-8-428-S2.xls]

\section{Additional file 3}

Core set of modulated genes during berry ripening. $\log _{2}$ average expression data for 2003, 2005 and 2006 of the 1477 modulated genes during berry ripening. Some more information such as the corresponding TC or singleton of the TIGR gene index $v 4.0$, the expression profile cluster, the sequence annotation and the corresponding GO functional category are also included.

Click here for file

[http://www.biomedcentral.com/content/supplementary/1471-

2164-8-428-S3.xls]

\section{Additional file 4}

List of the GO functional categories. Gene Ontology codes with obsolete and updated descriptions of the functional categories used in this work. Click here for file

[http://www.biomedcentral.com/content/supplementary/14712164-8-428-S4.xls]

\section{Additional file 5}

Functional categories distribution in the Vitis GeneChip ${ }^{\varpi}$. Comparison of the functional categories distribution in the Affymetrix Vitis GeneChip and in the Arabidopsis genome.

Click here for file

[http://www.biomedcentral.com/content/supplementary/1471-

2164-8-428-S5.tiff]

\section{Additional file 6}

Functional categories distribution in the expression clusters. Functional categories distribution in the eight clusters obtained by the $k$-means method on the gene expression profiles of the 1477 modulated genes. Click here for file

[http://www.biomedcentral.com/content/supplementary/14712164-8-428-S6.tiff]

\section{Additional file 7}

Complete list of genes belonging to the functional categories discussed in the article. Genes of the core set involved in the regulation of berry development (part A) and responsible for berry phenotypic traits (part B), ordered by major GO functional categories (in red). For clarity, transcripts discussed in the manuscript were further classified according to GO more specific terms.

Click here for file

[http://www.biomedcentral.com/content/supplementary/14712164-8-428-S7.xls]

\section{Additional file 8}

List of the primers used for the RT-PCR validation experiment. Sequence of the primers used in real time reverse transcription-polymerase chain reaction.

Click here for file

[http://www.biomedcentral.com/content/supplementary/14712164-8-428-S8.doc]

\section{Acknowledgements}

We wish to thank Dr. Davide Marchignoli for informatic support and stimulating discussion, Dr. Massimo Bertamini, Dr. Roberto Zorer for physiological data on berry samples and seasonal climate data (IASMA Research Center), Dr. Mauro Dallaserra (FBK \& CNR-Istituto di Biofisica, TrentoItaly) for $\mathrm{H}_{2} \mathrm{O}_{2}$ measurements, Dr. Etti Or (Volcani Center, Israel) and Dr. David Neale (University of California, Davis - USA) for critical reading of the manuscript and Dr. Enrico Blanzieri for help with data analysis.

This work was supported in part by grants from the Fondo Unico of the Provincia Autonoma di Trento, post doc project "Profiles" (S.P.), and from the Advanced Biology Project funded by the Fondazione delle Casse di Risparmio di Trento e Rovereto.

\section{References}

I. Doligez A, Adam-Blondon AF, Cipriani G, Di Gaspero G, Laucou V, Merdinoglu D, Meredith CP, Riaz S, Roux C, This P: An integrated SSR map of grapevine based on five mapping populations. Theor Appl Genet 2006, I I 3:369-382.

2. IASMA Genomics [http://genomics.research.iasma.it]

3. NCBI dbest [http://www.ncbi.nlm.nih.gov/dbEST/]

4. Coombe BG, Mccarthy MG: Dynamics of Grape Berry Growth and Physiology of Ripening. Aust J Grape Wine Res 2000, 6:131-135. 
5. Ablett E, Seaton G, Scott K, Shelton D, Graham MW, Baverstock P, Lee LS, Henry R: Analysis of grape ESTs: global gene expression patterns in leaf and berry. Plant Sci 2000, I59:87-95.

6. Moser C, Segala C, Fontana P, Salakhudtinov I, Gatto P, Pindo M, Zyprian E, Toepfer R, Grando MS, Velasco R: Comparative analysis of expressed sequence tags from different organs of Vitis vinifera L. Funct Integr Genomics 2005, 5:208-2I7.

7. da Silva FG, Iandolino A, AI-Kayal F, Bohlmann MC, Cushman MA, Lim $H$, Ergul A, Figueroa R, Kabuloglu EK, Osborne C, Rowe J, Tattersal E, Leslie A, Xu J, Baek J, Cramer GR, Cushman JC, Cook DR: Characterizing the grape transcriptome. Analysis of expressed sequence tags from multiple Vitis species and development of a compendium of gene expression during berry development. Plant Physiol 2005, 139:574-597.

8. Davies C, Robinson SP: Differential screening indicates a dramatic change in mRNA profiles during grape berry ripening. Cloning and characterization of CDNAs encoding putative cell wall and stress response proteins. Plant Physiol 2000, I 22:803-8|2.

9. Waters DL, Holton TA, Ablett EM, Lee LS, Henry RJ: cDNA microarray analysis of developing grape (Vitis vinifera cv. Shiraz) berry skin. Funct Integr Genomics 2005, 5:40-58.

10. Terrier N, Glissant D, Grimplet J, Barrieu F, Abbal P, Couture C, Ageorges A, Atanassova R, Leon C, Renaudin JP, Dedaldechamp F, Romieu C, Delrot S, Hamdi S: Isogene specific oligo arrays reveal multifaceted changes in gene expression during grape berry (Vitis vinifera L.) development. Planta 2005, 222:832-847.

II. Grimplet J, Deluc LG, Tillett RL, Wheatley MD, Schlauch KA, Cramer GR, Cushman JC: Tissue-specific mRNA expression profiling in grape berry tissues. BMC Genomics 2007, 8:187.

12. Coombe BG: Adoption of a system for identifying grapevine growth stages. Aust J Grape Wine Res 1995, I: 104-1 I0.

13. Tusher VG, Tibshirani R, Chu G: Significance analysis of microarrays applied to the ionizing radiation response. Proc Natl Acad Sci USA 200I, 98:5I I6-5I2I.

14. Gene Ontology home [http://www.geneontology.org/]

15. TAIR:The Arabidopsis Information Resource [http://www.ara bidopsis.org]

16. Cercos M, Soler G, Iglesias DJ, Gadea J, Forment J, Talon M: Globa analysis of gene expression during development and ripening of citrus fruit flesh. A proposed mechanism for citric acid utilization. Plant Mol Biol 2006, 62:5। 3-527.

17. Alba R, Payton P, Fei Z, McQuinn R, Debbie P, Martin GB, Tanksley SD, Giovannoni JJ: Transcriptome and selected metabolite analyses reveal multiple points of ethylene control during tomato fruit development. Plant Cell 2005, 17:2954-2965.

18. Coombe BG, Hale CR: The hormone content of ripening grape berries and the effects of growth substance treatments. Plant Physiol 1 973, 5 1:629-634.

19. Davies C, Boss PK, Robinson SP: Treatment of grape berries, a nonclimacteric fruit with a synthetic auxin, retards ripening and alters the expression of developmentally regulated genes. Plant Physiol 1997, I I 5: I 155- I I6I.

20. Aharoni A, Keizer LC, Van Den Broeck HC, Blanco-Portales R Munoz-Blanco J, Bois G, Smit P, De Vos RC, O'Connell AP: Novel insight into vascular, stress, and auxin-dependent and -independent gene expression programs in strawberry, a non-climacteric fruit. Plant Physiol 2002, 1 29:1019-1031.

21. Gillaspy G, Ben-David H, Gruissem W: Fruits: a developmental perspective. Plant Cell 1993, 5:|439-I45I.

22. Cawthon DL, Morris JR: Relationship of seed number and maturity to berry development, fruit maturation, hormonal changes, and uneven berry ripening in 'Concord' (Vitis labrusca) grapes. J Am Soc Hortic Sci 1982: I097- I 104.

23. Symons GM, Davies C, Shavrukov Y, Dry IB, Reid JB, Thomas MR: Grapes on steroids. Brassinosteroids are involved in grape berry ripening. Plant Physiol 2006, I40:I50- I58.

24. Pollmann S, Neu D, Weiler EW: Molecular cloning and characterization of an amidase from Arabidopsis thaliana capable of converting indole-3-acetamide into the plant growth hormone, indole-3-acetic acid. Phytochemistry 2003, 62:293-300.

25. Staswick PE, Serban B, Rowe M, Tiryaki I, Maldonado MT, Maldonado MC, Suza W: Characterization of an Arabidopsis enzyme family that conjugates amino acids to indole-3-acetic acid. Plant Cell 2005, 17:616-627.
26. Liu K, Kang BC, Jiang H, Moore SL, Li H, Watkins CB, Setter TL, Jahn MM: A GH3-like gene, CcGH3, isolated from Capsicum chinense $\mathrm{L}$. fruit is regulated by auxin and ethylene. Plant Mol Biol 2005, 58:447-464.

27. Hale CR, Coombe BG, Hawker JS: Effects of ethylene and 2-chloroethylphosphonic acid on the ripening of grapes. Plant Physiol 1970, 45:620-623.

28. Trainotti L, Pavanello A, Casadoro G: Different ethylene receptors show an increased expression during the ripening of strawberries: does such an increment imply a role for ethylene in the ripening of these non-climacteric fruits? J Exp Bot 2005, 56:2037-2046.

29. Chervin C, El-Kereamy A, Roustan JP, Latche A, Lamon J, Bouzayen $M$ : Ethylene seems required for the berry development and ripening in grape, a non-climacteric fruit. Plant Sci 2004 167:1301-1305

30. Tournier B, Sanchez-Ballesta MT, Jones B, Pesquet E, Regad F, Latche $A$, Pech JC, Bouzayen M: New members of the tomato ERF family show specific expression pattern and diverse DNA-binding capacity to the GCC box element. FEBS Lett 2003, 550:149-154.

31. Lorenzo O, Piqueras R, Sanchez-Serrano Jj, Solano R: ETHYLENE RESPONSE FACTORI integrates signals from ethylene and jasmonate pathways in plant defense. Plant Cell 2003, I5:165-178.

32. Ohme-Takagi M, Shinshi H: Ethylene-inducible DNA binding proteins that interact with an ethylene-responsive element. Plant Cell 1995, 7: I73-182.

33. Xu P, Narasimhan ML, Samson T, Coca MA, Huh GH, Zhou J, Martin GB, Hasegawa PM, Bressan RA: A nitrilase-like protein interacts with GCC box DNA-binding proteins involved in ethylene and defense responses. Plant Physiol I 998, I I 8:867-874.

34. Zegzouti H, Jones B, Frasse P, Marty C, Maitre B, Latch A, Pech JC, Bouzayen $M$ : Ethylene-regulated gene expression in tomato fruit: characterization of novel ethylene-responsive and ripening-related genes isolated by differential display. Plant J 1999, 18:589-600.

35. Kneissl ML, Deikman J: The tomato E8 gene influences ethylene biosynthesis in fruit but not in flowers. Plant Physiol 1996, I I 2:537-547.

36. Aharoni A, O'Connell AP: Gene expression analysis of strawberry achene and receptacle maturation using DNA microarrays. J Exp Bot 2002, 53:2073-2087.

37. Giovannoni J]: Genetic regulation of fruit development and ripening. Plant Cell 2004, I6(Suppl):SI70-SI80.

38. Zeevaart JAD, Creelman RA: Metabolism and physiology of abscisic-acid. Annu Rev Plant Physiol Plant Mol Biol 1988, 39:439-473.

39. Xiao $H$, Nassuth A: Stress- and development-induced expression of spliced and unspliced transcripts from two highly similar dehydrin I genes in V. riparia and V. vinifera. Plant Cell Rep 2006, 25:968-977.

40. Jang JY, Kim DG, Kim YO, Kim JS, Kang H: An expression analysis of a gene family encoding plasma membrane aquaporins in response to abiotic stresses in Arabidopsis thaliana. Plant Mol Biol 2004, 54:7। 3-725.

4I. Montoya T, Nomura T, Yokota T, Farrar K, Harrison K, Jones JD, Kaneta T, Kamiya Y, Szekeres M, Bishop GJ: Patterns of Dwarf expression and brassinosteroid accumulation in tomato reveal the importance of brassinosteroid synthesis during fruit development. Plant J 2005, 42:262-269.

42. Nakamichi N, Murakami-Kojima M, Sato E, Kishi Y, Yamashino T Mizuno T: Compilation and characterization of a novel WNK family of protein kinases in Arabiodpsis thaliana with reference to circadian rhythms. Biosci Biotechnol Biochem 2002, 66:2429-36

43. Kikis EA, Khanna R, Quail PH: ELF4 is a phytochrome-regulated component of a negative-feedback loop involving the central oscillator components CCAI and LHY. Plant J 2005, 44:300-313.

44. Kardailsky I, Shukla VK, Ahn JH, Dagenais N, Christensen SK, Nguyen JT, Chory J, Harrison MJ, Weigel D: Activation tagging of the floral inducer FT. Science 1999, 286: 1962-1965.

45. Hwang I, Chen HC, Sheen J: Two-component signal transduction pathways in Arabidopsis. Plant Physiol 2002, I 29:500-5I5.

46. Ageorges A, Fernandez L, Vialet S, Merdinoglu D, Terrier N, Romieu $C$ : Four specific isogenes of the anthocyanin metabolic path- 
way are systematically co-expressed with the red colour of grape berries. Plant Sci 2006, 170:372-383.

47. Kobayashi S, Ishimaru M, Hiraoka K, Honda C: Myb-related genes of the Kyoho grape (Vitis labruscana) regulate anthocyanin biosynthesis. Planta 2002, 21 5:924-933.

48. Deluc L, Barrieu F, Marchive C, Lauvergeat V, Decendit A, Richard T, Carde JP, Merillon JM, Hamdi S: Characterization of a grapevine R2R3-MYB transcription factor that regulates the phenylpropanoid pathway. Plant Physiol 2006, 140:499-5II.

49. Boss PK, Buckeridge EJ, Poole A, Thomas MR: New insights into grapevine flowering. Funct Plant Biol 2003, 30:593-606.

50. Boss PK, Vivier M, Matsumoto S, Dry IB, Thomas MR: A cDNA from grapevine (Vitis vinifera $L$.), which shows homology to AGAMOUS and SHATTERPROOF, is not only expressed in flowers but also throughout berry development. Plant Mol Biol 200I, 45:54I-553.

51. Vrebalov J, Ruezinsky D, Padmanabhan V, White R, Medrano D, Drake R, Schuch W, Giovannoni J: A MADS-box gene necessary for fruit ripening at the tomato ripening-inhibitor (rin) locus. Science 2002, 296:343-346.

52. Olsen AN, Ernst HA, Leggio LL, Skriver K: NAC transcription factors: structurally distinct, functionally diverse. Trends Plant $\mathrm{SC}$ 2005, 10:79-87.

53. Ulker B, Somssich IE: WRKY transcription factors: from DNA binding towards biological function. Curr Opin Plant Biol 2004, 7:49|-498.

54. Paquette AJ, Benfey PN: Maturation of the ground tissue of the root is regulated by gibberellin and SCARECROW and requires SHORT-ROOT. Plant Physiol 2005, 138:636-640.

55. Cakir B, Agasse A, Gaillard C, Saumonneau A, Delrot S, Atanassova $R$ : A grape ASR protein involved in sugar and abscisic acid signaling. Plant Cell 2003, I5:2165-2I80.

56. Atanassova R, Leterrier M, Gaillard C, Agasse A, Sagot E, CoutosThevenot $P$, Delrot S: Sugar-regulated expression of a putative hexose transport gene in grape. Plant Physiol 2003, I 3 I:326-334.

57. Sarry JE, Sommerer N, Sauvage FX, Bergoin A, Rossignol M, Albagnac G, Romieu C: Grape berry biochemistry revisited upon proteomic analysis of the mesocarp. Proteomics 2004, 4:20I-2I 5 .

58. Robinson SP, Davies C: Molecular biology of grape berry ripening. Aust / Grape Wine Res 2000, 6: I75-I88.

59. Nunan KJ, Davies C, Robinson SP, Fincher GB: Expression patterns of cell wall-modifying enzymes during grape berry development. Planta 200।, 2 I 4:257-264.

60. Saladie M, Rose JK, Cosgrove DJ, Catala C: Characterization of a new xyloglucan endotransglucosylase/hydrolase $(X T H)$ from ripening tomato fruit and implications for the diverse modes of enzymic action. Plant J 2006, 47:282-295

61. Jimenez A, Creissen G, Kular B, Firmin J, Robinson S, Verhoeyen M, Mullineaux $P$ : Changes in oxidative processes and components of the antioxidant system during tomato fruit ripening. Planta 2002, 2 | 4:75। -758.

62. Meir S, Philosoph-Hadas S, Zauberman G, Fuchs Y, Akerman M, Aharoni $\mathrm{N}$ : Increased formation of fluorescent lipid-peroxidation products in avocado peels precedes other signs of ripening. $J$ Amer Soc Hort Sci I 99I, I I 6:823-826.

63. Moyle R, Fairbairn DJ, Ripi J, Crowe M, Botella JR: Developing pineapple fruit has a small transcriptome dominated by metallothionein. J Exp Bot 2005, 56:101-II2.

64. Brennan T, Frenkel C: Involvement of hydrogen peroxide in the regulation of senescence in pear. Plant Physiol 1977, 59:4 I I-4I6.

65. Mittler R, Vanderauwera S, Gollery M, Van Breusegem F: Reactive oxygen gene network of plants. Trends Plant Sci 2004, 9:490-498.

66. Ogawa T, Ueda Y, Yoshimura K, Shigeoka S: Comprehensive analysis of cytosolic Nudix hydrolases in Arabidopsis thaliana. J Biol Chem 2005, 280:25277-25283.

67. Terrier N, Romieu C: Grape berry acidity. Kluwer Academic Publisher; $2001 \cdot 35-57$

68. Cheng GW, Breen PJ: Activity of phenylalanine ammonia-lyase (PAL) and concentrations of anthocyanins and phenolics in developing strawberry fruit. I Am Soc Hortic Sci |991, I 1 6:865-869.

69. Zhang X-Y, Wang X-L, Wang X-F, Xia G-H, Pan Q-H, Fan R-C, Wu $\mathrm{F}-\mathrm{Q}$, Yu X-C, Zhang D-P: A shift of phloem unloading from symplasmic to apoplasmic pathway is involved in developmenta onset of ripening in grape berry. Plant Physiol 2006, I 42:220-232.
70. Davies C, Wolf T, Robinson SP: Three putative sucrose transporters are differentially expressed in grapvine tissues. Plant Sci 1999, 147:93-100.

71. Davies C, Robinson SP: Sugar accumulation in grape berries. Cloning of two putative vacuolar invertase CDNAs and their expression in grapevine tissues. Plant Physiol 1996, I I I:275-283.

72. Nguyen-Quoc B, Foyer $\mathrm{CH}$ : A role for 'futile cycles' involving invertase and sucrose synthase in sucrose metabolism of tomato fruit. J Exp Bot 200I, 52:88I-889.

73. Terrier N, Sauvage FX, Ageorges A, Romieu C: Changes in acidity and in proton transport at the tonoplast of grape berries during development. Planta 200I, 213:20-8.

74. Mellema S, Eichenberger W, Rawyler A, Suter M, Tadege M, Kuhlemeier $C$ : The ethanolic fermentation pathway supports respiration and lipid biosynthesis in tobacco pollen. Plant / 2002, 30:329-336.

75. Boss PK, Davies C, Robinson SP: Analysis of the expression of anthocyanin pathway genes in developing Vitis vinifera $\mathrm{L}$. $\mathrm{Cv}$ Shiraz grape berries and the implications for pathway regulation. Plant Physiol 1996, I I I: 1059-1066.

76. Martinoia E, Klein M, Geisler M, Bovet L, Forestier C, Kolukisaoglu U, Muller-Rober B, Schulz B: Multifunctionality of plant ABC transporters - more than just detoxifiers. Planta 2002, 2 I 4:345-355

77. Wissel K, Pettersson F, Berglund A, Jansson S: What affects mRNA levels in leaves of field-grown aspen? A study of developmental and environmental influences. Plant Physiol 2003, I33: I190-II97.

78. Salome PA, McClung CR: PSEUDO-RESPONSE REGULATOR 7 and 9 are partially redundant genes essential for the temperature responsiveness of the Arabidopsis circadian clock. Plant Cell 2005, I7:79I-803.

79. Ulm R, Baumann A, Oravecz A, Mate Z, Adam E, Oakeley E], Schafer $E$, Nagy F: Genome-wide analysis of gene expression reveals function of the bZIP transcription factor HY5 in the UV-B response of Arabidopsis. Proc Natl Acad Sci USA 2004, IOI:1397-I402

80. Tepperman JM, Zhu T, Chang HS, Wang X, Quail PH: Multiple transcription-factor genes are early targets of phytochrome $A$ signaling. Proc Natl Acad Sci USA 200I, 98:9437-9442.

8I. Conde C, Agasse A, Glissant D, Tavares R, Geros H, Delrot S: Pathways of glucose regulation of monosaccharide transport in grape cells. Plant Physiol 2006, I4I:1563-I577.

82. Fillion L, Ageorges A, Picaud S, Coutos-Thevenot P, Lemoine R, Romieu $C$, Delrot $S$ : Cloning and expression of a hexose transporter gene expressed during the ripening of grape berry. Plant Physiol 1999, I 20: 1083-1094.

83. Vignault C, Vachaud M, Cakir B, Glissant D, Dedaldechamp F, Buttner M, Atanassova R, Fleurat-Lessard P, Lemoine R, Delrot S: VvHTI encodes a monosaccharide transporter expressed in the conducting complex of the grape berry phloem. J Exp Bot 2005, 56: $|409-14| 8$

84. Goto-Yamamoto N, Wan GH, Masaki K, Kobayashi S: Structure and transcription of three chalcone synthase genes of grapevine (Vitis vinifera). Plant Sci 2002, 162:867-872.

85. Harmer SL, Hogenesch JB, Straume M, Chang HS, Han B, Zhu T, Wang X, Kreps JA, Kay SA: Orchestrated transcription of key pathways in Arabidopsis by the circadian clock. Science 2000 , 290:2II0-2II3

86. Moser C, Gatto P, Moser M, Pindo M, Velasco R: Isolation of functional RNA from small amounts of different grape and apple tissues. Mol Biotechnol 2004, 26:95-100.

87. Gentleman RC, Carey VJ, Bates DM, Bolstad B, Dettling M, Dudoit S, Ellis B, Gautier L, Ge Y, Gentry J, Hornik K, Hothorn T, Huber W, lacus S, Irizarry R, Leisch F, Li C, Maechler M, Rossini AJ, Sawitzki G, Smith C, Smyth G, Tierney L, Yang JY, Zhang J: Bioconductor: open software development for computational biology and bioinformatics. Genome Biol 2004, 5:R80.

88. Ihaka R, Gentleman R: R: a language for data analysis and graphics. J Comput Graph Stat 1996, 5:299-314.

89. Wu Z, Irizarry RA: Preprocessing of oligonucleotide array data. Nat Biotechno 2004, 22:656-658. author reply 658

90. TM4: Microarray software suite [http://www.tm4.org/]

91. Affymetrix home site [http://www.affymetrix.com/index.affx]

92. Nadershahi A, Fahrenkrug SC, Ellis LB: Comparison of computational methods for identifying translation initiation sites in EST data. BMC Bioinformatics 2004, 5: 14-. 
93. ESTate [http://www.bio.net/bionet/mm/bio-soft//999-December/ 02I776.html]

94. Altschul SF, Madden TL, Schaffer AA, Zhang J, Zhang Z, Miller W, Lipman DJ: Gapped BLAST and PSI-BLAST: a new generation of protein database search programs. Nucleic Acids Res 1997, 25:3389-3402.

95. Ramakers C, Ruijter JM, Deprez RH, Moorman AF: Assumptionfree analysis of quantitative real-time polymerase chain reaction (PCR) data. Neurosci Lett 2003, 339:62-66.

96. Pfaffl MW: A new mathematical model for relative quantification in real-time RT-PCR. Nucleic Acids Res 200I, 29:e45.

97. ArrayExpress home [http://www.ebi.ac.uk/microarray-as/aer/ ?\#ae-main[0]]

Publish with Biomed Central and every scientist can read your work free of charge

"BioMed Central will be the most significant development for disseminating the results of biomedical research in our lifetime. "

Sir Paul Nurse, Cancer Research UK

Your research papers will be:

- available free of charge to the entire biomedical community

- peer reviewed and published immediately upon acceptance

- cited in PubMed and archived on PubMed Central

- yours - you keep the copyright

Submit your manuscript here:

http://www.biomedcentral.com/info/publishing_adv.asp
BioMedcentral 